\title{
BOUQUETS OF CIRCLES FOR LAMINATION LANGUAGES AND COMPLEXITIES
}

\author{
Philippe NARBEL ${ }^{1}$
}

\begin{abstract}
Laminations are classic sets of disjoint and non-selfcrossing curves on surfaces. Lamination languages are languages of twoway infinite words which code laminations by using associated labeled embedded graphs, and which are subshifts. Here, we characterize the possible exact affine factor complexities of these languages through bouquets of circles, i.e. graphs made of one vertex, as representative coding graphs. We also show how to build families of laminations together with corresponding lamination languages covering all the possible exact affine complexities.
\end{abstract}

Mathematics Subject Classification. 14Q05, 37B10, 37F20, 57R30, 68R15, 68Q45, 68R10.

\section{INTRODUCTION}

Laminations on surfaces are closed sets of pairwise disjoint one-dimensional submanifolds (the lamination leaves) which can be considered as curves with no preferred parameterization $[9,28]$. The notion of lamination generalizes the notion of foliation of surfaces, i.e. global decomposition of surfaces into one-dimensional submanifolds, and can also be seen as a way of considering singular foliations, i.e. foliations defined everywhere except at a finite number of points [6]. Laminations occur for instance as fixed subsets of surface diffeomorphisms. A usual technique to study laminations in surface theory is to deform them continuously into embedded graphs, often in the form of train tracks [25,28], but also in the form of more general graphs. Laminations are then said to be carried by these graphs.

Keywords and phrases. Curves, laminations on surfaces, symbolic dynamics, shifts, factor complexity, embedded graphs, train-tracks, Rauzy graphs, substitutions, spirals.

1 LaBRI - UFR Math-Info, University of Bordeaux 1, 33405 Talence, France.

narbel@labri.fr 
When such carrier graphs are labeled, the involved curves inherit the labels of the paths they are deformed into, giving rise to lamination languages, which are languages made of two-way infinite words, and which happen to be specific subshifts (or shifts) [19]. Lamination languages can indeed be looked at as subshifts of edge-shifts [17] constrained by the geometry of the set of curves they represent.

With this relationship in mind between geometry and formal language theory, the main purpose of this paper is to give some results about how the notion of lamination helps to produce languages with specific properties, and also conversely, how languages with their associated tools help to describe laminations.

Our focus here is on a classic word combinatorics notion: the (factor) complexity of a language $L$ of infinite words is the function $p_{L}(n)$ over $\mathbb{N}^{*}$, where for each $n, p_{L}(n)$ is the number of factors (or subblocks) of length $n$ occurring in the words in $L[8,22]$. In particular, this complexity definition is the basic ingredient of the topological entropy of $L$, defined as $\lim _{n \rightarrow \infty} \log \left(p_{L}(n)\right) / n$ [1]. Lamination languages are instances of languages with zero entropy as their complexities are always ultimately affine, that is, of the form $a n+b, \forall n>n_{0}$, for some $n_{0} \geq 0$. With this respect, we shall here characterize what are the possible forms of their exact affine complexities, i.e. when $n_{0}=0$ :

Theorem 1.1. A lamination language $L$ with an exact complexity $p_{L}$ is such that $p_{L}(n)=$ an $+b, \forall n>0$, with $(a, b) \in \mathbb{N} \times \mathbb{Z}$, and $b \geq\left\lceil-\frac{a}{2}+1\right\rceil$. Conversely, for every $p_{L}$ satisfying the preceding conditions, there exist lamination languages with this complexity.

Note that a consequence of this result is that the exact complexities of lamination languages do not cover all the exact affine complexities that can take shifts [7].

The proof of Theorem 1 will rely on the fact that there is no univocal relationship between laminations, carrier graphs and lamination languages, giving thus some freedom to transform the last two while geometrically preserving laminations. In particular, by applying edge contractions to carrier graphs (closely related to usual Whitehead moves for singular foliations), one can turn these graphs into bouquets of circles, i.e. graphs made of a single vertex and $m \geq 1$ edges. These elementary graphs happen to be generic enough to describe all the possible exact complexities of lamination languages. A coherent bouquet of $m$ circles, i.e. an embedded bouquet with its single vertex having all its incoming (resp. outgoing) edges consecutive around it, carries laminations which correspond to the dynamics of interval exchange transformations on $m$ intervals, that is, orientationpreserving and piecewise isometric maps of bounded intervals [5]. As a matter of fact, lamination languages include the natural symbolic representations of interval exchanges, known to have affine complexity of the form $p(n)=(m-1) n+1$ [16], and thus also include Sturmian languages, which have an exact affine complexity $p(n)=n+1[20,23]$. Non-coherent bouquets of circles play then an important role for producing all the affine complexities given by Theorem 1.1, and accordingly this paper develops and extends the tools introduced in [19] to deal with non-coherent graphs. 
Next, as a complement to the converse part of Theorem 1.1, we show how embedded bouquets of circles can be used to explicitly construct lamination languages for each possible exact complexity, yielding at the same time a technique to build laminations, in particular laminations with a finite number of curves and connected as sets. The main result with this respect will be the following:

Theorem 1.2. There exist families of lamination languages made of ultimately periodic words, having exact complexities an $+b, \forall n>0$, covering all the possible $a$ and $b$ 's given by Theorem 1.1, and coding finite connected laminations.

For infinite lamination languages we still do not know about a constructive method to obtain a family of them covering all the possible complexities. Here we just present how to obtain some of these languages from pseudo-Anosov surface diffeomorphisms, i.e. transformations leaving two laminations fixed, one stable and the other one unstable. Some of these surface transformations [24, 29] are indeed known to translate into the symbolic domain as substitutions, whose fixed points are representatives of the lamination languages coding the corresponding stable laminations $[18,19]$. Contracting the involved carrier graphs produces minimal infinite languages associated with bouquets of circles too, sometimes non-coherent ones.

\section{BASIC DEFINITIONS}

\subsection{Curves, laminations And Graphs}

We begin with some definitions of geometric-oriented notions, some of them being in a simplified form but sufficient for the text (for more detailed ones, the reader may refer to $[5,6,9,28])$. A surface $\Sigma$ is a two-dimensional manifold. A surface of finite type is a closed surface from which finitely many points, called punctures, have been removed. When endowed with a complete Riemannian metric with constant curvature -1 a surface is said to be hyperbolic. The objects under study here mainly belong to hyperbolic surface theory, and $\Sigma$ will henceforth always denote an oriented surface of finite type with some fixed hyperbolic metric (whose choice does not play any role in this paper). A curve $\gamma$ in $\Sigma$ is a continuous map, either from a closed connected subset $J \subseteq \mathbb{R}$, or from the circle $S^{1}$ to $\Sigma$. In the latter case $\gamma$ is said to be closed (and also in the former case when the map is periodic). If the map is injective, $\gamma$ is said to be simple. If $J=\mathbb{R}, \gamma$ is said to be two-way infinite, and if $J$ is bounded and $\gamma$ is simple, then $\gamma$ is called an arc.

Let $\Gamma$ be a finite directed graph embedded in $\Sigma$. An admissible path in $\Gamma$ is a sequence of consecutive edges with the same orientation. For the sake of simplicity, we henceforth assume that for every vertex $v$ of $\Gamma$, its indegree $\partial^{-}(v)$ and outdegree $\partial^{+}(v)$ are strictly positive, that is, $v$ is crossed by at least one admissible path ${ }^{2}$. A curve $\gamma$ in $\Sigma$ is said to be carried by $\Gamma$ if it can be continuously

\footnotetext{
${ }^{2}$ This simplification has no effect on the generality of the results of the paper. Vertices with $\partial^{-}(v)=0$ or $\partial^{+}(v)=0$ could be included by making them correspond to punctures of the surface, but this adds no new combinatorial behavior to the considered curves.
} 
deformed into an admissible path of $\Gamma$, by a free homotopy if $\gamma$ is closed, or by a uniformly continuous homotopy if $\gamma$ is two-way infinite [5]. The next figure shows a closed curve $\gamma$ carried by a graph $\Gamma$ where $\Sigma$ is a torus of genus 2 with one puncture:

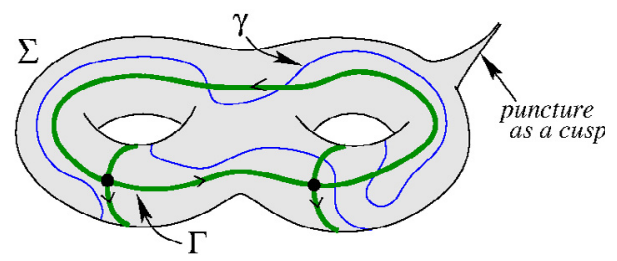

In order to exclude the carrying of ill-behaved curves, $\Gamma$ is always assumed to be embedded in such a way that, among the connected components of $\Sigma \backslash \Gamma$ there is no disk bounded by a cycle of $\Gamma$ with less than two punctures. A set of curves is said to be carried by $\Gamma$ if all its curves are carried by $\Gamma$.

In a general setting, a lamination is a foliation of a closed subset of $\Sigma$, that is, roughly, a decomposition into one-dimensional submanifolds of this subset [28]. The laminations mostly used in surface theory are geodesic laminations, i.e. those made of geodesics only $[9,28]$. These are equivalent up to isotopy to laminations made of pairwise non-homotopic curves, and deformable to graphs ([28], 8.9.4). Here, the laminations we consider are always of this kind, and we use an alternative definition of them up to isotopy, also coming from Thurston and related to the preceding equivalence, which is essentially the following [19]: a (topological) lamination $\mathcal{L}$ in $\Sigma$ is a set of simple closed or two-way infinite curves in $\Sigma$, all pairwise disjoint and non-homotopic, such that there exists an embedded graph $\Gamma$ which carries $\mathcal{L}$ in a maximal way with respect to inclusion (no other curve carried by $\Gamma$ can be added to $\mathcal{L}$ while preserving the curve set properties) ${ }^{3}$.

Simple examples of laminations are given by the sets of pairwise disjoint and non-homotopic simple closed curves on $\Sigma$. These are finite laminations, i.e. laminations made of a finite number of curves. An instance is shown on the left of the next figure, made of $\gamma_{1}, \gamma_{2}, \gamma_{3}$, and carried by $\Gamma$ consisting of three disjoint cycles:
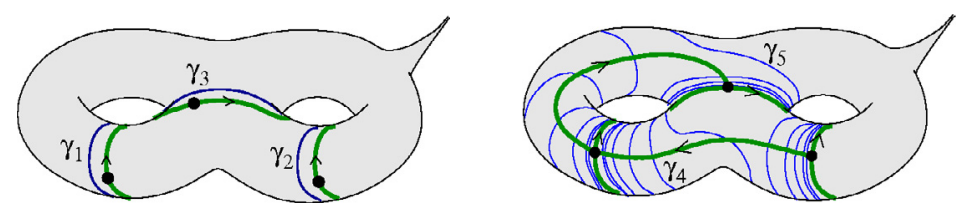

Other examples of finite laminations are obtained by using infinite curves spiraling along simple closed curves [5]. An instance is shown on the right of the figure above,

\footnotetext{
3 The graphs we consider here are directed - so as to more easily code laminations -, implying that laminations are assumed orientable, whereas for the general case one classically uses specific non-directed graphs, generally train tracks $[25,28]$. However in our context, non-orientable laminations can be considered as being carried by directed double graph covers to which all admissible paths lift, reflecting the fact that an admissible path may go through an edge in both directions $[19,28]$. Thus there is no loss of generality in considering directed graphs only.
} 
where two spiraling curves $\gamma_{4}, \gamma_{5}$ have been added to the lamination shown on the left, while two edges have been added to $\Gamma$ to carry these curves.

Examples of infinite laminations are classically obtained via interval exchange transformations [21] which are, up to scaling, orientation-preserving and piecewise isometries of $I=[0,1)$. Such a map $T: I \rightarrow I$ can be seen as permuting a finite number of semi-open subintervals $I_{1}, \ldots, I_{m}$ partitioning $I$. More precisely, $T$ is determined by $(\lambda, \pi)$, where $\lambda=\left[\lambda_{1}, \ldots, \lambda_{m}\right]$ is a probability vector made of the $I_{i}$ 's lengths in their order of occurrence in $I$, and $\pi$ is a permutation of $\{1, \ldots, m\}$, so that the effect of $T$ is to concatenate the $I_{i}$ 's in its image in the order given by $\pi$, the vector of lengths becoming $\left[\lambda_{\pi^{-1}(1)}, \ldots, \lambda_{\pi^{-1}(m)}\right]$. For instance, here is a representation of an interval exchange over 3 intervals, with $\pi=\left(\begin{array}{lll}1 & 2 & 3\end{array}\right)$ :

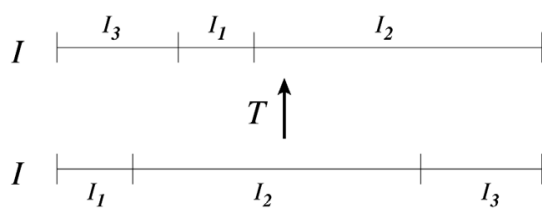

A lamination can be obtained from an interval exchange $T$ given by $(\lambda, \pi)$ using its suspension [5]. Let $R=[0,1] \times[0, \delta]$, for some $\delta>0$, be a closed rectangle corresponding to $I$, foliated by the $\operatorname{arcs} x \times[0, \delta]$, and let $S^{\text {down }}=[0,1] \times 0$ and $S^{u p}=[0,1] \times \delta$ be its sides of length 1 . For each $I_{i}$ of $T$, let $R_{i}=\left[0, \lambda_{i}\right] \times[0,1]$ be a closed rectangle foliated by the arcs $x \times[0,1]$, with $S_{i}^{\text {down }}, S_{i}^{u p}$ its sides of length $\lambda_{i}$. Next, the $S_{i}^{\text {down' }}$ 's are identified to $S^{\text {up }}$ in the same order as $I_{1}, \ldots, I_{m}$, with their ends as the only intersections, and the $S_{i}^{u p}$ 's to $S^{\text {down }}$ in the order given by $\pi$, i.e. $I_{\pi^{-1}(1)}, \ldots, I_{\pi^{-1}(m)}$, so that the result is an orientable band-like surface $\Sigma_{T}$ which is covered with pairwise disjoint curves made of identified arcs from the rectangle identifications. For instance, considering $T$ as in the above example, its corresponding $\Sigma_{T}$ is shown on the left of the next figure:
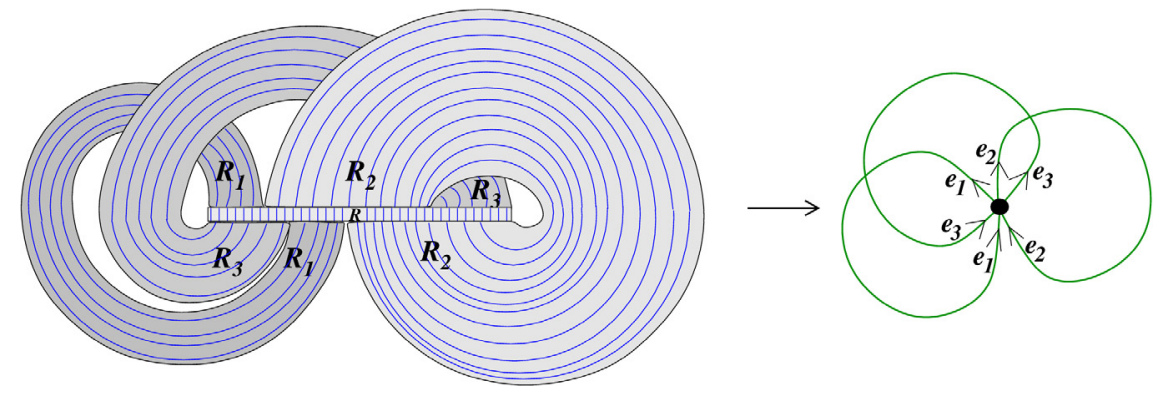

By glueing a punctured disk along each boundary component of $\Sigma_{T}$, we get a surface $\Sigma$ of finite type. Then, by slitting out the induced singular curves, i.e. the curves starting or ending at the intersections between the $R_{i}$ 's sides, and by keeping only one curve from each set of pairwise homotopic curves, a lamination $\mathcal{L}_{T}$ is obtained on $\Sigma[5,15]$. This lamination is carried by a bouquet of $m$ circles $\Gamma_{T}$ embedded in $\Sigma$, i.e. a graph made of a single vertex and $m$ edges, described here 
by placing a vertex $v$ in $R$, and by defining one edge $e_{i}$ for each $R_{i}$, linking $v$ to itself by going through this $R_{i}$ (see the example in the figure above). A vertex of an embedded directed graph is said to be coherent if all its incident incoming edges are consecutive around it using either cyclic order - hence its outgoing edges are consecutive too. An embedded directed graph is coherent if all its vertices are coherent. By the above construction, $\Gamma_{T}$ is a coherent bouquet of circles.

A lamination is said to be minimal if it does not contain any lamination as a proper non-empty subset; it is said to be aperiodic minimal if it is not reduced to a single closed curve. Similarly, an interval exchange transformation $T$ given by $(\lambda, \pi)$ is said to be minimal if for all $x \in I$, the full orbit $\left\{T^{n}(x)\right\}_{n \in \mathbb{Z}}$ is dense in $I$. A sufficient condition for minimality of $T$, called the infinite distinct orbit condition ( $i d o c$ for short), is that the orbits of all the $m-1$ points $x \in I$ of $T$ such that $x=\overline{I_{i}} \cap \overline{I_{i+1}}$, with $i \in[1, \ldots, m-1]$, are infinite and disjoint [16]. The idoc is satisfied if $\pi$ is irreducible and if the only rational relation between the $\lambda_{i}$ 's in $\lambda$ is $\sum_{i} \lambda_{i}=1$. The lamination $\mathcal{L}_{T}$ is aperiodic minimal iff $T$ is minimal. Thus we can exhibit infinite laminations through interval exchanges satisfying the idoc. Note that the maximality condition used in the lamination definition is not necessary for a lamination $\mathcal{L}$ to be carried by a graph $\Gamma$, but when it holds, we say that $\mathcal{L}$ is maximal rel. to $\Gamma$. For instance, if an interval exchange $T$ satisfies the idoc then the associated lamination $\mathcal{L}_{T}$ is maximal rel. to its bouquet of circles $\Gamma_{T}$ [19].

Even more examples of laminations can be obtained by using the fact that the union of finitely many minimal sublaminations with finitely many two-way infinite curves whose ends spiral along the minimal sublaminations is a lamination [5].

\subsection{Coding Laminations}

Let $A$ be a finite alphabet. Let $A^{\mathbb{Z}}$ denote the set of the two-way infinite words over $A$. A directed graph $\Gamma$ is here said to be labeled by $A$ if its edges are bijectively labeled by $A$. The label of an admissible path of $\Gamma$ is the word obtained by concatenating the labels of its edges. If $\gamma$ is a curve carried by $\Gamma$, and if it is homotopic to a unique path, its coding is the label of this path. In this case, we also say that $\gamma$ is coded by this label, or coded by $\Gamma$.

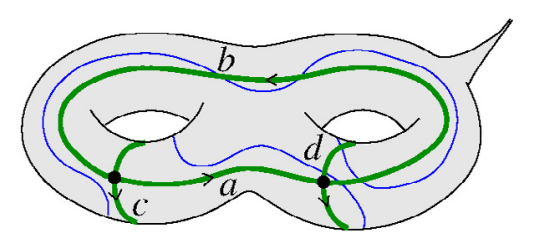

The coding of a carried closed curve $\gamma$ is the two-way infinite periodic word ${ }^{\omega} u^{\omega}$, where $u$ is the label of the closed path in $\Gamma$ freely homotopic to $\gamma$. The above figure shows a closed curve coded by ${ }^{\omega}(a d b c)^{\omega}$ on a punctured torus of genus 2 . Thus closed and two-way infinite curves are coded over $A^{\mathbb{Z}}$. By extension, a set of curves is said to be coded by $\Gamma$ if all its curves are coded by $\Gamma$. 
A language is a set of finite and/or infinite words [20,26]. In particular, a language in $A^{\mathbb{Z}}$ is a language of two-way infinite words. The full language $A^{\mathbb{Z}}$ can be endowed with the topology coming from the Cantor metric, i.e. for $w=$ $\ldots a_{-1} a_{0} a_{1} \ldots$ and $w^{\prime}=\ldots a_{-1}^{\prime} a_{0}^{\prime} a_{1}^{\prime} \ldots$ in $A^{\mathbb{Z}}$, with $a_{i}, a_{i}^{\prime} \in A$, their distance is 0 if they are equal, and $2^{-k}$ if they are not, where $k$ is the smallest non-negative integer for which $a_{k} \neq a_{k}^{\prime}$ or $a_{-k} \neq a_{-k}^{\prime}$. The shift map $\sigma$ on $A^{\mathbb{Z}}$ is the continuous transformation which sends $\ldots a_{-1} a_{0} a_{1} \ldots$ to $\ldots a_{-1}^{\prime} a_{0}^{\prime} a_{1}^{\prime} \ldots$ where $a_{i}^{\prime}=a_{i+1}$ for $i \in \mathbb{Z}$. A shift (or shift space or subshift) [17] is a closed $\sigma$-invariant language in $A^{\mathbb{Z}}$. The shift orbit closure $L^{\sigma}$ of a language $L$ in $A^{\mathbb{Z}}$ is the smallest shift which includes $L$. A lamination language is the shift orbit closure in $A^{\mathbb{Z}}$ of the codings of all the curves of a lamination $\mathcal{L}$ coded by a graph $\Gamma$ labeled by $A$; the shiftinvariance reflects the fact that the curves of a lamination are considered up to homotopy, hence up to parameterization, and the closure property is a consequence of the lamination definition (mainly, the maximality property) [19]. A lamination word is an infinite word in a lamination language.

For instance, from the example of the last figure, $\left\{\omega(a d b c)^{\omega}\right\}^{\sigma}$ is a simple lamination language. Examples of non-trivial lamination languages can be obtained via interval exchange transformations. Indeed, given an interval exchange $T: I \rightarrow I$, and a map cod $: I \rightarrow A$ assigning a distinct letter to each $I_{i}$ of the partition of $I$, the symbolic orbit of any $x \in I$ is the word $w_{T}(x)=\ldots \operatorname{cod}\left(T^{-1}(x)\right) \operatorname{cod}(x) \operatorname{cod}(T(x)) \ldots$ The symbolic orbit language of $T$ is the shift orbit closure of the language $\left\{w_{T}(x) \mid x \in I\right\}$, and it corresponds to the lamination language which codes the lamination $\mathcal{L}_{T}$ by $\Gamma_{T}$, built from $T$ in the preceding section.

\subsection{FACTOR COMPLEXITY}

A factor (or subblock) of a word $w$ is a finite word $u$ such that $w=w^{\prime} u w^{\prime \prime}$, where $w^{\prime}, u, w^{\prime \prime}$ are words $\left(w^{\prime}, w^{\prime \prime}\right.$ being possibly empty words). The set of all the distinct factors of a word $w$ is denoted by Fact $_{w}$, and for a language $L$, by Fact $_{L}=\bigcup_{w \in L}$ Fact $_{w}$. The set of all the distinct length- $n$ factors of a word $w$ is denoted by $\operatorname{Fact}_{w}(n)$, and for a language $L$, by $\operatorname{Fact}_{L}(n)=\bigcup_{w \in L} \operatorname{Fact}_{w}(n)$. An infinite word is minimal (or uniformly recurrent) if each of its factors occurs infinitely often in it with bounded gaps. A shift is minimal if it has no proper non-empty subset as a shift. In a minimal shift $L$, every word $w \in L$ is such that $L={\overline{\left\{\sigma^{k}(w)\right\}}}_{k \in \mathbb{Z}}$, and for every $w, w^{\prime} \in L$, Fact $_{w}=$ Fact $_{w^{\prime}}$, even if $w, w^{\prime}$ are only half-words in $L$. Since a minimal lamination is coded by a minimal shift $L$, its combinatorics can thus be studied through a single (half-)word in $L$.

The (factor) complexity $[8,22]$ of a word $w$ is the function $p_{w}: \mathbb{N}^{*} \rightarrow \mathbb{N}^{*}$, where $p_{w}(n)=\left|F_{a c t}(n)\right|$, i.e. the cardinality of Fact $_{w}(n)$. The complexity of a language $L$ is defined as $p_{L}(n)=\left|\operatorname{Fact}_{L}(n)\right|$. Accordingly, if $L \subset A^{\mathbb{Z}}$ is a minimal shift, every word $w \in L$ is such that $p_{w} \equiv p_{L}$. A complexity formula is said to 
be ultimate if there exists $n_{0} \geq 0$, such that it holds for all $n>n_{0}$, and it is also said to be exact when $n_{0}=0$. Here are two known results about affine complexity:

Theorem 2.1 (see [7], 5.3). Let $(a, b) \in \mathbb{N} \times \mathbb{Z}$. Then there is a word $w$ of exact affine complexity $p_{w}(n)=a n+b, \forall n>0$, iff $a+b \geq 1$ and $2 a+b \leq(a+b)^{2}$.

Theorem 2.2 (see [19], B). A lamination language $L$ is such that there exist $n_{0} \geq 0, a \in \mathbb{N}, b \in \mathbb{Z}$, so that $p_{L}(n)=a n+b, \forall n>n_{0}$.

The purpose of the next two sections is to prove Theorem 1.1, that is, mainly to precise Theorem 2.2 for the exact complexity case in similar terms to Theorem 2.1.

\section{GRAPHS AND BURSTS}

\subsection{LINE GRAPHS AND LANGUAGES}

Let $\Gamma=(V, E)$ be a finite directed graph where $V$ denotes the set of vertices, and $E$ the set of edges. The line graph of $\Gamma$ is the directed graph $S(\Gamma)=\left(V_{S}, E_{S}\right)$, where $V_{S}=E$, and $E_{S}$ is such that there is an edge from $e_{i}$ to $e_{j}$ if the sequence $e_{i} e_{j}$ corresponds to a length-2 admissible path in $\Gamma$.
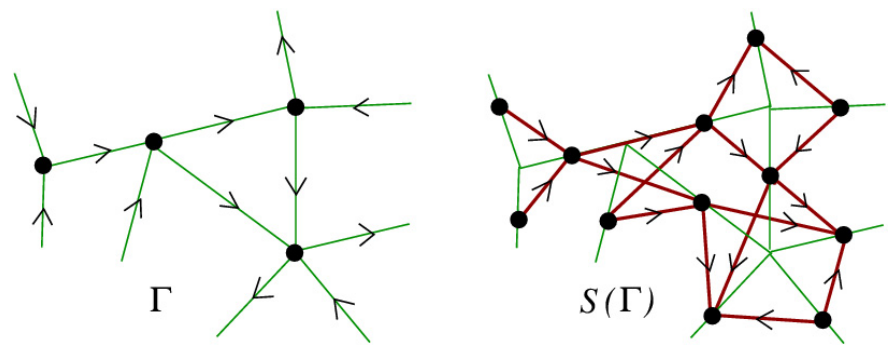

When defined from an embedded graph $\Gamma$ in $\Sigma$, the graph $S(\Gamma)$ inherits an induced immersion in $\Sigma$ (not necessarily an embedding since edge crossings may occur):

(i) Each vertex of $S(\Gamma)$ is placed in the interior of its corresponding edge of $\Gamma$.

(ii) The pair of vertices lying in a length-2 admissible path of $\Gamma$ are linked by an arc in $\Gamma$ which is contained in this length-2 path.

(iii) The arcs defined in (ii) are put in general and minimal intersection position in $\Sigma$ with their end vertices fixed (see the figure above).

The graph $S(\Gamma)$ will henceforth always be considered with this induced immersion.

Now, assume $\Gamma$ has been labeled by $A$, and let $L$ be a language in $A^{\mathbb{Z}}$ made of labels of admissible paths in $\Gamma$, that is, $\operatorname{Fact}_{L}(2)$ is a set of labels of length-2 admissible paths in $\Gamma$. Let us also assume that every letter of $A$ is used in $L$, that is, Fact $_{L}(1)=A$. We then define the graph $S_{L}(\Gamma)$ as $S(\Gamma)$ in which we keep only the edges corresponding to edge pairs of $\Gamma$ labeled by Fact $_{L}(2)$. Accordingly, $S_{L}(\Gamma)$ 
inherits the immersion of $S(\Gamma)$. The graph $S_{L}(\Gamma)$ is also related to a classic representation of $F_{a c t}(n)$, usually called the $n$-th order Rauzy graph of $L$, i.e. the directed graph where each vertex corresponds to a distinct factor in Fact $_{L}(n)$, and where an edge between two vertices $a u, u b$, with $a, b \in A, u \in \operatorname{Fact}_{L}(n-1)$, exists iff $a u b \in \operatorname{Fact}_{L}(n+1)[12]:$

Lemma 3.1 (see [19], Sect. 3). Let $\Gamma$ be a graph embedded in $\Sigma$, and let $L$ be a language in $A^{\mathbb{Z}}$ of labels of admissible paths in $\Gamma$. Then:

(1) $S_{L}(\Gamma)$ is isomorphic to the first-order Rauzy graph of $L$.

(2) $S_{L}(\Gamma)$ is a coherent graph.

When $L$ is a lamination language, $S_{L}(\Gamma)$ comes with additional properties:

Lemma 3.2 (see [19], Sect. 3). Let $\Gamma$ be a graph embedded in $\Sigma$ carrying a lamination $\mathcal{L}$, and let $L$ be the lamination language coding $\mathcal{L}$ by $\Gamma$. Then:

(1) $S_{L}(\Gamma)$ is an embedding (not just an immersion).

(2) $S_{L}(\Gamma)$ still carries $\mathcal{L}$.

(3) A curve carried by $S_{L}(\Gamma)$ is carried by $\Gamma$ too.

(4) If $\mathcal{L}$ is maximal rel. to $\Gamma$, it is also maximal rel. to $S_{L}(\Gamma)$.

For instance, here is again a torus of genus 2, with an embedded graph $\Gamma$ labeled by $\{a, b, c, d\}$, so that $S(\Gamma)$ is made of the edges labeled by $\{a b, a d, b a, b c, c a, c c, d b, d d\}$; the drawn carried disjoint curves are coded into a language $L$, so that $S_{L}(\Gamma)$ is embedded, with edges corresponding to $\operatorname{Fact}_{L}(2)=$ $\{a b, a d, b a, b c, c a, d b\}$ :

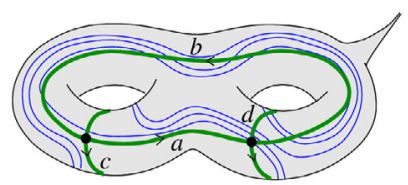

$\Gamma$

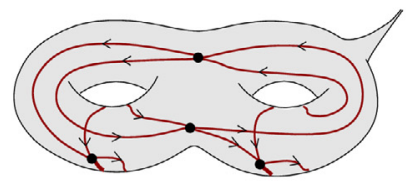

$S(\Gamma)$

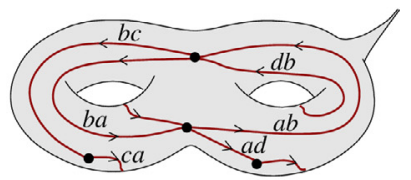

$S_{L}(\Gamma)$

\subsection{Bursts AND OUTERPLANAR GRAPHS}

When building $S_{L}(\Gamma)$ from $\Gamma$, each vertex $v$ of $\Gamma$ is transformed into a subgraph in $S_{L}(\Gamma)$, called a burst of $v$, whose vertices correspond to the edges of $\Gamma$ incident with $v$, and whose edges correspond to the length-2 admissible paths labeled by Fact $_{L}(2)$ and going through $v$. Such a burst can be represented by a bipartite graph Burst $_{v, L}(\Gamma)=\left(V_{v \text {,in }} \sqcup V_{v \text {,out }}, E_{v}\right)$, where the vertices in $V_{v \text {,in }}$ correspond to the incoming half-edges at $v$, denoted by $v_{i}^{-}$, where the vertices in $V_{v \text {, out }}$ correspond to the outgoing half-edges at $v$, denoted by $v_{i}^{+}$, and where $E_{v}$ is the set of edges which correspond to length-2 admissible paths labeled by $F_{a c t}(2)$ and going through one incoming and one outgoing half-edge of $v$. Burst ${ }_{v, L}(\Gamma)$ is directed too, its 
edge orientations going from $V_{v \text {,in }}$ to $V_{v \text {,out }}$. Here is an example at some vertex $v$ for some fixed $L$ for which $F_{a c t}(2)=\{a e, a f, b c, b e, d c, d e, f e, f f\}$, and where the vertices of Burst $_{v, L}(\Gamma)$ are $V_{v \text {,in }}=\left\{v_{a}^{-}, v_{b}^{-}, v_{d}^{-}, v_{f}^{-}\right\}, V_{v, \text { out }}=\left\{v_{c}^{+}, v_{e}^{+}, v_{f}^{+}\right\}$:

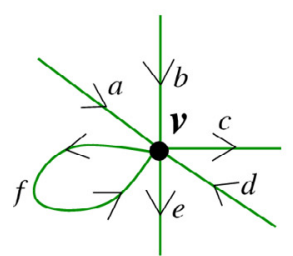

$\Gamma$

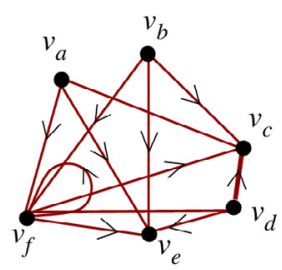

$S(\Gamma)$

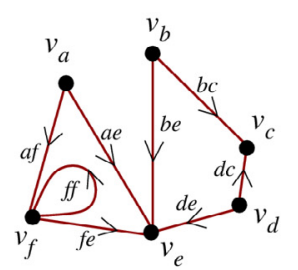

$S_{L}(\Gamma)$

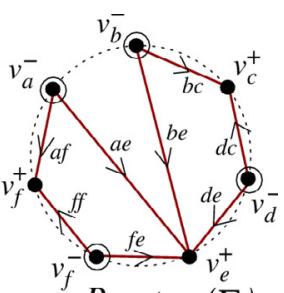

Burst $_{v, L}(\Gamma)$

As this example shows, Burst $_{v, L}(\Gamma)$ is not always isomorphic to the burst it represents in $S_{L}(\Gamma)$ since it relies on the incident half-edges at $v$, thus any loop at $v$ makes two distinct vertices in Burst $_{v, L}(\Gamma)$. Still, Burst $_{v, L}(\Gamma)$ has also its edges in correspondence with $\operatorname{Fact}_{L}(2)$, and it is more convenient to work with. In addition, when defined from an immersed $S_{L}(\Gamma)$ in $\Sigma$, Burst ${ }_{v, L}(\Gamma)$ has an induced immersion (up to isotopy):

(i) Let $D \subset \Sigma$ be a disk containing $v$ in its interior, such that its boundary $\partial D$ intersects only the incident half-edges of $v$. Then each vertex in $V_{v \text {,in }}$ and $V_{v \text {,out }}$ is placed at the intersection of its corresponding half-edge and $\partial D$.

(ii) Each edge of Burst $_{v, L}(\Gamma)$ links the corresponding vertices in $V_{v \text {,in }}$ to the ones in $V_{v \text {,out }}$ by a straight $\operatorname{arc}$ within $D$.

In the figure above, Burst $_{v, L}(\Gamma)$ is shown with its immersion. Note that given some Burst $_{v, L}(\Gamma)$, in order to get the subgraph it represents in $S_{L}(\Gamma)$, one has just to identify each pair $\left(v_{i}^{-}, v_{i}^{+}\right)$coming from a loop at $v$, by dragging $v_{i}^{-}$to $v_{i}^{+}$ along this same loop.

Here is a full example where $\Gamma$ is a non-coherent bouquet of three circles with its single vertex $v$, where its embedding surface $\Sigma$ can be the sphere with as many punctures as needed so that $\Gamma$ is a coding carrier graph, that is, a graph ensuring unique curve carrying. We then consider two disjoint curves in $\Sigma$ carried by $\Gamma$, and coded by $L=\left\{{ }^{\omega} b c a^{\omega},{ }^{\omega} b(c a)^{\omega}\right\}$, so that $F_{a c t}(2)=\{a a, a c, b b, b c, c a\}$. Thus Burst ${ }_{v, L}(\Gamma)$ has five edges, from which $S_{L}(\Gamma)$, being itself the burst of $v$, is obtained by identifying $v_{i}^{-}$and $v_{i}^{+}$, for each $i=a, b, c$, along their corresponding loop $i$ to get $v_{i}$ :

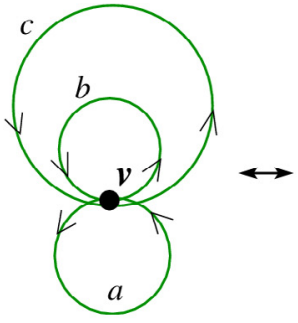

$\Gamma$

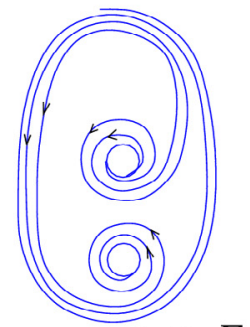

curves carried by $\Gamma$

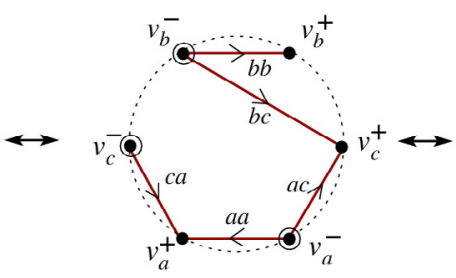

Burst $_{v, L}(\Gamma)$

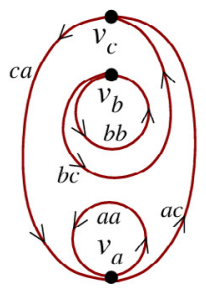

$S_{L}(\Gamma)$ 
A (planar) drawing of a graph is an embedding of this graph in the plane; here by extension, in a surface $\Sigma$, it is an embedding of this graph in a disk in $\Sigma$ possibly punctured. A drawing is outerplanar if all its vertices belong to a single face, and a graph is outerplanar if it admits an outerplanar drawing.

Lemma 3.3. Let $\Gamma$ be a graph embedded in $\Sigma$. Let $L$ be a language in $A^{\mathbb{Z}}$ of labels of admissible paths in $\Gamma$ such that $S_{L}(\Gamma)$ is embedded too. Then, for every vertex $v \in \Gamma$, the induced immersion of Burst ${ }_{v, L}(\Gamma)$ in $\Sigma$ is an outerplanar drawing in $\Sigma$.

Proof. Since $S_{L}(\Gamma)$ is embedded, the burst at any vertex $v \in \Gamma$ has edges corresponding to Fact $_{L}(2)$ which do not cross with each other. Since $\operatorname{Burst}_{v, L}(\Gamma)$ reflects how the arcs corresponding to these edges cross a small disk $D$ containing $v$, its immersion in $\Sigma$ is a graph drawing. The vertices of $\operatorname{Burst}_{v, L}(\Gamma)$ can belong to $\partial D$, while its edges are included in $D$, hence the outerplanarity.

Thus from now on, when coming from an embedded $S_{L}(\Gamma)$ in $\Sigma$, e.g. when $L$ is a lamination language, Burst $_{v, L}(\Gamma)$ will always be considered with its outerplanar drawing. Also, a property $\mathcal{P}$ of a graph or a drawing will be said to be maximal if one cannot add any edge to it while preserving $\mathcal{P}$.

Proposition 3.4 (see [13]). A drawing of a bipartite graph $\left(V_{1} \sqcup V_{2}, E\right)$ where $\left|V_{1}\right| \leq\left|V_{2}\right|$, which is maximally outerplanar, has at most $2\left|V_{1}\right|+\left|V_{2}\right|-2$ edges.

In order to exhibit outerplanar drawings with the maximal number of edges, given a set of vertices $\left(V_{1} \sqcup V_{2}\right)$ of a bipartite graph with $\left|V_{1}\right| \leq\left|V_{2}\right|$, put these vertices on a circle while maximizing the alternations between the vertices of $V_{1}$ and $V_{2}$. The vertices of $V_{1}$ can then occur between vertices of $V_{2}$, and they can thus be linked by arcs to their neighbors, which makes $2\left|V_{1}\right|$ edges. Next, pick any vertex of $V_{1}$, which can be linked to all the other vertices of $V_{2}$ within the circle, which makes $\left|V_{2}\right|-2$ more edges, hence a total of $2\left|V_{1}\right|+\left|V_{2}\right|-2$ edges.

Note that with respect to the embedding of $\Gamma$ in $\Sigma$ and to a burst of a vertex $v$ of $\Gamma$, the above maximal alternation of the vertices in $V_{1}=V_{v \text {,in }}$ and $V_{2}=V_{v \text {,out }}$ corresponds to a maximal alternation of the orientations of the incident incoming and outgoing half-edges of $v$, that is, to a "maximal non-coherence" at $v$. For instance, in the example to the left of the figure below, the vertex $v$ is alternating, i.e. its incident half-edges show a strict alternation of their orientations, and $L$ can be chosen so that the maximal number of edges of the corresponding $\operatorname{Burst}_{v, L}(\Gamma)$ is attained, i.e. ten edges (here, $\left|V_{v, \text { in }}\right|=\partial^{-}(v)=4$ and $\left|V_{v \text {, out }}\right|=\partial^{+}(v)=4$ ):
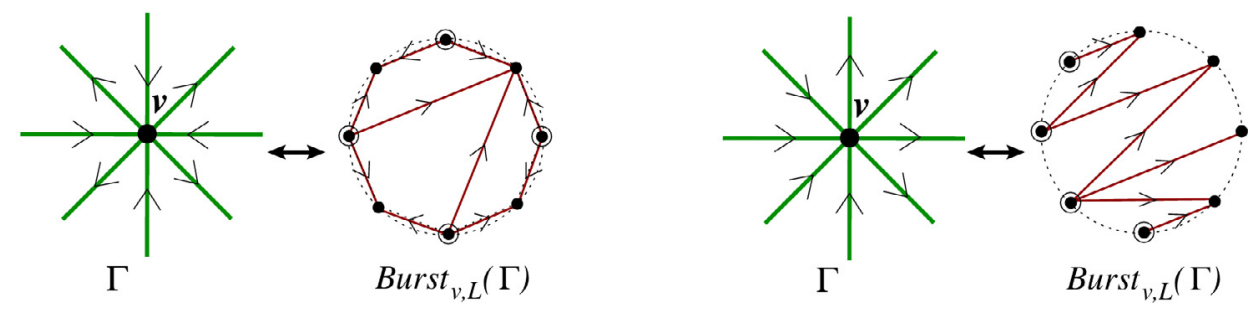
To the right of the figure above is shown a case where there is a minimum orienta-

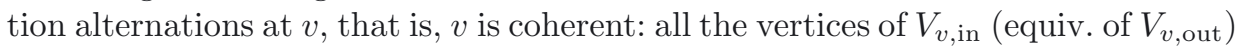
of the corresponding Burst $_{v, L}(\Gamma)$ are consecutive around the circle. This kind of outerplanar drawing is also said to be biplanar, i.e. $V_{v}$,in and $V_{v \text {, out }}$ can also be respectively placed in two parallel lines, the edges remaining straight arcs. A graph admitting such a drawing is known to be a union of caterpillar trees [10], i.e. trees such that deleting all their leaves yield linear path graphs. In the above example, the maximal number of edges of $\operatorname{Burst}_{v, L}(\Gamma)$ is attained for such a biplanar graph, that is, as a non-directed graph, it is a single caterpillar tree of seven edges.

When $L$ is a lamination language, $\operatorname{Burst}_{v, L}(\Gamma)$ has some specific properties:

Lemma 3.5. Let $\Gamma$ be a graph embedded in $\Sigma$, carrying a lamination $\mathcal{L}$, and let $L$ be the lamination language coding $\mathcal{L}$ by $\Gamma$. Then for every vertex $v \in \Gamma$ (recall that $\left|V_{v, \text { in }}\right|=\partial^{-}(v)$ and $\left.\left|V_{v, \text { out }}\right|=\partial^{+}(v)\right)$ :

(1) The number of edges of Burst ${ }_{v, L}(\Gamma)$ belongs to the interval $\left[k_{\max }, 2 k_{\min }+\right.$ $\left.k_{\max }-2\right]$, where $k_{\min }=\min \left(\partial^{-}(v), \partial^{+}(v)\right)$ and $k_{\max }=\max \left(\partial^{-}(v), \partial^{+}(v)\right)$.

(2) Let Burst,$L(\Gamma)$ be a maximal embedding, that is, no edge can be added to it while preserving the embedding. Then it is connected (as a non-directed graph), and it has at least $\partial^{-}(v)+\partial^{+}(v)-1$ edges (the minimum being attained when $v$ is coherent, thus Burst ${ }_{v, L}(\Gamma)$ is biplanar).

(3) Let $\mathcal{L}$ be a lamination maximal rel. to $\Gamma$. Then for every vertex $v \in \Gamma$, Burst $_{v, L}(\Gamma)$ is a maximal embedding.

Proof.

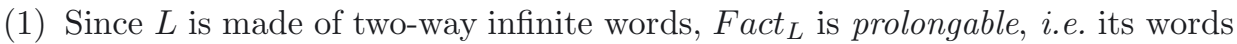
can be prolongated by one letter at least in one way to the right and to the left so that these prolongations remain in Fact ${ }_{L}$. Thus, since the edges in Burst $_{v, L}(\Gamma)$ correspond to edges in $S_{L}(\Gamma)$ labeled by words in $F_{a c t}(2)$, there is no isolated vertex in Burst $_{v, L}(\Gamma)$, which has then at least $k_{\max }$ edges. Moreover, since $L$ is a lamination language, $S_{L}(\Gamma)$ is embedded, then Lemma 3.3 applies, and Burst $_{v, L}(\Gamma)$ is outerplanar, hence by Proposition 3.4 the result follows.

(2) If $\operatorname{Burst}_{v, L}(\Gamma)$ is a maximal embedding, it is maximally outerplanar. Assume then that it is made of two disjoint outerplanar subgraphs $\Gamma_{1}$ and $\Gamma_{2}$ (the case where there are $n$ of them is handled similarly). Let $F$ denote the external face of $\Gamma$, which is also the external face of $\Gamma_{1}$ intersected with the external face of $\Gamma_{2}$. Let $D$ be the disk whose boundary contains the vertices of $\operatorname{Burst}_{v, L}(\Gamma)$, and let $F^{\prime}$ be the component region in $D \cap F$. Since Burst $_{v, L}(\Gamma)$ is disconnected without any isolated vertex, $F^{\prime}$ contains in its boundary at least one edge of $\Gamma_{1}$ and one edge in $\Gamma_{2}$. Also, since $\operatorname{Burst}_{v, L}(\Gamma)$ is bipartite, these two edges link vertices of $V_{v \text {,in }}$ to vertices of $V_{v \text {, out }}$. But then one edge, linking a vertex 
of $V_{v \text {,in }}$ to a vertex of $V_{v \text {,out }}$, can be added within $F^{\prime}$ while preserving planarity, contradicting maximality. Hence as a non-directed graph, $\operatorname{Burst}_{v, L}(\Gamma)$ is connected, so it is at least a tree. This situation happens when $\operatorname{Burst}_{v, L}(\Gamma)$ is maximally biplanar, being then a caterpillar tree with $\partial^{-}(v)+\partial^{+}(v)-1$ edges.

(3) If we could add one edge $e$ to Burst $_{v, L}(\Gamma)$ while preserving the embedding, a distinct curve carried by $\Gamma$ could be built from $e$, and added to $\mathcal{L}$ while preserving the overall properties of the resulting set of curves ([19], Sect. 3.3.3).

An embedded bouquet of circles is alternating if its single vertex is alternating.

Corollary 3.6. Let $\Gamma$ be a bouquet of $m$ circles embedded in $\Sigma$, carrying a lamination $\mathcal{L}$, and let $L$ be the lamination language coding $\mathcal{L}$ by $\Gamma$. Then for the vertex $v$ of $\Gamma$, the number of edges of Burst ${ }_{v, L}(\Gamma)$ (and thus of $S_{L}(\Gamma)$ too) belongs to $[m, 3 m-2]$ (the maximum being attained when $\Gamma$ is alternating, and Burst $_{v, L}(\Gamma)$ is maximally outerplanar).

Proof. For a bouquet of circles $\Gamma$, the number of edges of Burst $_{v, L}(\Gamma)$ is the same as for $S_{L}(\Gamma)$. Since $\partial^{-}(v)=\partial^{+}(v)=k_{\min }=k_{\max }=m$, Lemma 3.5(1) gives the interval $[m, 3 m-2]$. Using the outerplanar drawing construction introduced after Proposition 3.4, a strict alternation of the half-edges incident with $v$ allows the maximum of edges given by Proposition 3.4 to be attained.

\section{COMPlexity AND LAMinations}

\subsection{TOOLS FOR COMPUTING COMPLEXITY}

From now on, $\Gamma$ will always denote a lamination carrier graph embedded in $\Sigma$. Given a lamination coded into $L$ by $\Gamma$, the role of $S_{L}(\Gamma)$ for computing the complexity of $L$ comes from the following result, keeping in mind that by Lemma 3.1(1), $S_{L}(\Gamma)$ is the first-order Rauzy graph of $L$ (so that $|A|$ is the number of its vertices, and $\mid$ Fact $_{L}(2) \mid$ is the number of its edges, which depends on the bursts of $\Gamma$ ):

Proposition 4.1 (see [19], 4.1.2). Let $L$ be a lamination language coding a lamination $\mathcal{L}$ by a graph $\Gamma$ labeled by $A$. Then $L$ has an exact affine complexity $p_{L}$ iff $\mathcal{L}$ is maximal rel. to $S_{L}(\Gamma)$. Moreover:

$$
p_{L}(n)=\left(\mid \text { Fact }_{L}(2)|-| A \mid\right) n+\left(2|A|-\mid \text { Fact }_{L}(2) \mid\right), \quad \forall n>0 .
$$

Note that this result also applies if $\mathcal{L}$ is maximal rel. to $\Gamma$, since according to Lemma 3.2(4), $\mathcal{L}$ must be then maximal rel. to $S_{L}(\Gamma)$ too. Now, the formula above is not an invariant for laminations, as the same lamination can be carried by many different graphs. With this respect, there exist simple graph moves over a graph $\Gamma$ (closely related to Whitehead moves for singular foliations [6]), which preserve the carrying of a lamination $\mathcal{L}$, while transforming the coding of $\mathcal{L}$. In other words, 
these moves can be used to transform a lamination language with a specific complexity into another one with another complexity. One such type of graph move is edge contraction: let $e$ be an edge linking two distinct vertices $v_{1}$ and $v_{2}$ in $\Gamma$, then the contraction of $e$ consists in erasing $e$ from $\Gamma$, and in replacing $e$ in the set of incident edges with $v_{1}$ by all the incident edges with $v_{2}$, using the same cyclic order. For instance:
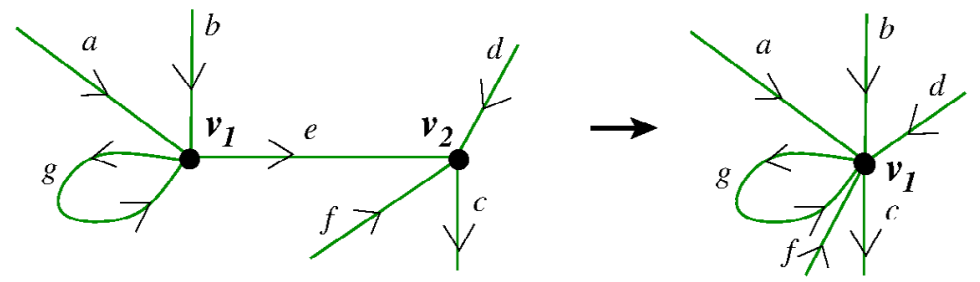

Lemma 4.2. Let $\mathcal{L}$ be a lamination coded by $\Gamma$ into $L$, and maximal rel. to $S_{L}(\Gamma)$. Let $\Gamma^{\prime}$ be $\Gamma$ to which one edge contraction has been applied, and let $L^{\prime}$ be the coding of $\mathcal{L}$ by $\Gamma^{\prime}$. Then $\mathcal{L}$ is also maximal rel. to $S_{L^{\prime}}\left(\Gamma^{\prime}\right)$.

Proof. Let $e$ be the contracted edge, and $v_{e}$ be its corresponding vertex in $S_{L}(\Gamma)$, which exists since $\operatorname{Fact}_{L}(1)$ is always assumed to be the labeling alphabet of $\Gamma$. Since $e$ is an edge between two distinct vertices of $\Gamma$, there is no loop incident with $v_{e}$ in $S_{L}(\Gamma)$. Thus such a contraction of $e$ means to erase $v_{e}$ from $S_{L}(\Gamma)$, and to replace it by the set of edges corresponding to every length-2 path going through $v_{e}$ and used to carry $\mathcal{L}$, so as to describe $S_{L^{\prime}}\left(\Gamma^{\prime}\right)$. But then, this transformation also corresponds to the burst of $v_{e}$ with respect to the language $L^{\prime \prime}$ which codes $\mathcal{L}$ by $S_{L}(\Gamma)$, that is, to Burst $_{v_{e}, L^{\prime \prime}}$. A consequence of Lemma 3.2(4) is that a burst preserves the maximality of a carried lamination, whence the result.

Lemma 4.3. Let $L$ be a lamination language coding a lamination $\mathcal{L}$ by a graph $\Gamma$, $\mathcal{L}$ being maximal rel. to $S_{L}(\Gamma)$. Let $\Gamma^{\prime}$ be $\Gamma$ to which one edge contraction has been applied, and let $L^{\prime}$ be the coding of $\mathcal{L}$ by $\Gamma^{\prime}$. Then, $p_{L^{\prime}}(n)=p_{L}(n)-1, \forall n>0$.

Proof. Let $L^{\prime \prime}$ be the language coding $\mathcal{L}$ by $S_{L}(\Gamma)$. In the proof of Lemma 4.2 , we saw that a contraction of $e$ in $\Gamma$ means to erase $v_{e}$ in $S_{L}(\Gamma)$, and to replace it by Burst $t_{v_{e}, L^{\prime \prime}}$, a transformation defining $S_{L^{\prime}}\left(\Gamma^{\prime}\right)$. Moreover, $\mathcal{L}$ is maximal rel. to $S_{L}(\Gamma)$, and by Lemma $3.1(2), S_{L}(\Gamma)$ is a coherent graph. By Lemma 3.5, Burst $_{v_{e}, L^{\prime \prime}}$ is thus a maximal biplanar drawing. Then, since there is no loop incident with $v_{e}$ in $S_{L}(\Gamma)$, the erasing of $v_{e}$ means to replace its $\partial^{-}\left(v_{e}\right)+\partial^{+}\left(v_{e}\right)$ incident edges by $\partial^{-}\left(v_{e}\right)+\partial^{+}\left(v_{e}\right)-1$ edges. By Lemma $4.2, \mathcal{L}$ is still maximal rel. to $S_{L^{\prime}}\left(\Gamma^{\prime}\right)$, thus Proposition 4.1 applies to obtain $p_{L^{\prime}}$, with $\left|F_{a c t} t_{L^{\prime}}(2)\right|=$ $\mid$ Fact $_{L}(2) \mid-1$, and with a labeling alphabet $A^{\prime}$ such that $\left|A^{\prime}\right|=|A|-1$.

\subsection{The EXACT COMPLEXITIES OF LAMINATION LANGUAGES}

A lamination carrier graph $\Gamma$ is said to be recurrent, if for every edge $e$ in $\Gamma$ there exists a simple closed curve which uses $e$ to be carried by $\Gamma$. When $\Gamma$ is coherent and recurrent, its edges can be weighted by maps like $\mu: E \rightarrow \mathbb{R}_{+}^{*}$ for which 
at every vertex $v$ of $\Gamma$ the branch equation $\sum_{i} \mu\left(e_{i}^{-}\right)=\sum_{j} \mu\left(e_{j}^{+}\right)$holds, where the $e_{i}^{-}$'s are the incoming incident edges at $v$, and the $e_{j}^{+}$'s the outgoing ones $[6,25]$. From these equations, a weighted coherent graph $\Gamma$ can be transformed into a band-like surface in a similar way to the construction of $\Sigma_{T}$ given in Section 2.1 for an interval exchange $T$ : each edge $e$ of $\Gamma$ is replaced by a foliated rectangle $R_{e}=[0, \mu(e)] \times[0,1]$, and these rectangles are glued together along their sides of length $\mu(e)$, reflecting the incidence patterns of the edges at each vertex of $\Gamma$.

Lemma 4.4. Let $\Gamma$ be a coherent and recurrent graph. Then there exist laminations carried and maximal rel. to $\Gamma$.

Proof. With the same treatment as for $\Sigma_{T}$, the above construction is known to yield laminations carried by $\Gamma[6,15,25]$. Next, if such a lamination is not maximal rel. to $\Gamma$, there always exists a finite set of curves carried by $\Gamma$ which is sufficient so that its union with $\mathcal{L}$ becomes a maximal lamination rel. to $\Gamma$ ([19], Sect. 3).

Now, recall that given a lamination language $L$, the graph $S_{L}(\Gamma)$ is determined by the bursts of $\Gamma$ 's vertices induced by $L$. Thus more abstractly, without any reference to a given $L$, a well-formed set of bursts $B(\Gamma)$ is a set of outerplanar drawings in $\Sigma$ of bipartite graphs without isolated vertex, one for each vertex $v \in \Gamma$, such that for the graph $\left(V_{1} \sqcup V_{2}, E\right)$ associated with $v$, we have $\left|V_{1}\right|=\partial^{-}(v)$, $\left|V_{2}\right|=\partial^{+}(v)$, and $V_{1} \sqcup V_{2}$ corresponds to the adjacent half-edges around $v$ on $\Gamma$, placed in the same cyclic ordering. The embedded graph $S_{B(\Gamma)}(\Gamma)$ is then obtained by replacing each $v \in \Gamma$ with its corresponding burst in $B(\Gamma)$.

Corollary 4.5. Let $\Gamma$ be a recurrent graph. Let $B(\Gamma)$ be a well-formed set of bursts. Then there exist laminations carried by $\Gamma$ and maximal rel. to $S_{B(\Gamma)}(\Gamma)$.

Proof. $\Gamma$ being recurrent, $S_{B(\Gamma)}(\Gamma)$ is recurrent too. From Lemma 3.1(2), one can also deduce $S_{B(\Gamma)}(\Gamma)$ is coherent, thus Lemma 4.4 applies to $S_{B(\Gamma)}(\Gamma)$. Then similarly to Lemma 3.2(3), to transform the bursts back into their vertices preserves the carrying, thus a lamination carried by $S_{B(\Gamma)}(\Gamma)$ is also carried by $\Gamma$.

We can now prove Theorem 1.1, that is, characterize what are the possible exact complexities for lamination languages:

Proof. Let $\Gamma$ be an alternating bouquet of $m$ circles with its single vertex $v$. A bouquet of circles is recurrent, and thus for any well-formed burst of $v$, denoted by Burst $_{v}$, making by itself a well-formed set $B(\Gamma)$, Corollary 4.5 ensures the existence of laminations carried by $\Gamma$ and maximal rel. to $S_{B(\Gamma)}(\Gamma)$.

According to Proposition 4.1, the coding $L$ of a lamination maximal rel. to $S_{L}(\Gamma)$ has complexity $p_{L}(n)=(K-m) n+(2 m-K), \forall n>0$, where $K$ is the number of edges of $S_{L}(\Gamma)$, equal to the number of edges of Burst $t_{v}$ since $\Gamma$ is a bouquet of circles. Next, by Corollary 3.6, $K \in[m, 3 m-2]$, and $\Gamma$ being alternating, this number of edges can take the maximal value, that is, there exists some Burst $_{v}$ with $3 m-2$ edges. This burst defines a well-formed $B(\Gamma)$, so that by the preliminary remark above, laminations carried by $\Gamma$ exist, and given any such lamination, 
its coding language $L$ satisfies Burst $_{v, L}(\Gamma)=$ Burst $_{v}$, and $S_{B(\Gamma)}(\Gamma)=S_{L}(\Gamma)$. Now, edges can be removed one by one from Burst $v$ until $m$ edges are left, so that at each edge removal the graph remains a burst defining a well-formed $B(\Gamma)$, and so that the same reasoning as before applies. Hence, $K$ can take every value in $[m, 3 m-2]$, and thus $p_{L}(n)=a n+b, \forall n>0$, where $a \in[0,2 m-2]$, and $b \in[2-m, m]$ with $b=m-a$, is a possible complexity for a lamination carried by $\Gamma$. In $\mathbb{N} \times \mathbb{Z}$, this set of pairs $(a, b)$ determines for each $m \geq 1$, a diagonal segment of slope -1 , starting from $(0, m)$ and going down to $(2 m-2,2-m)$, so as to cover an infinite region $Q=\left\{(a, b) \in \mathbb{N} \times \mathbb{Z} \mid a \geq 0, b \geq\left\lceil-\frac{a}{2}+1\right\rceil\right\}$ :

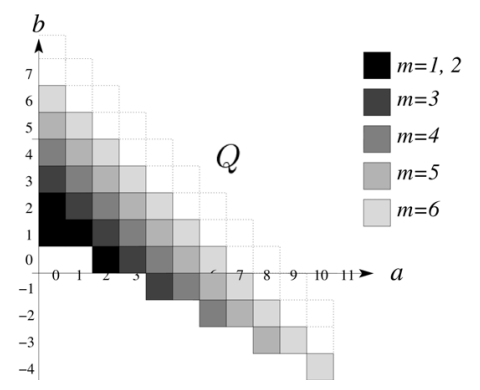

Hence laminations exist with coding languages having all the claimed complexities, and we have proved the converse part of Theorem 1.1.

Now, let $\Gamma$ be any embedded graph, that we first assume connected, and let $\mathcal{L}$ be a lamination carried by $\Gamma$ such that its coding lamination language $L_{0}$ has an exact complexity, that is, $p_{L_{0}}(n)=a_{0} n+b_{0}, \forall n>0$, for some $a_{0} \in \mathbb{N}, b_{0} \in \mathbb{Z}$. Then, according to Proposition $4.1, \mathcal{L}$ is maximal rel. to $S_{L_{0}}(\Gamma)$. We then contract the edges of $\Gamma$ one by one, and we get at each contraction a new language $L_{i}$ coding $\mathcal{L}$ by the resulting graph. By Lemma 4.2, the affine complexity remains exact, i.e. $p_{L_{i}}(n)=a_{i} n+b_{i}, \forall n>0$, for which by Lemma $4.3, a_{i}=a_{0}$ and $b_{i}=b_{i-1}-1$. Since $\Gamma$ is assumed connected, edge contractions can be applied, say $h$ of them, until we get a bouquet of circles. But then, again by Corollary 3.6 and the above arguments for the specific $K$ obtained, $p_{L_{h}}(n)=a_{h} n+b_{h}=a_{0} n+b_{0}-h$ is such that $\left(a_{h}, b_{h}\right) \in Q$. Now, if $(a, b) \in Q$, then $\left(a, b^{\prime}\right) \in Q$ for every integer $b^{\prime} \geq b$. Thus the complexity of $L_{0}$ is such that $\left(a_{h}, b_{h}+h\right)=\left(a_{0}, b_{0}\right) \in Q$.

In the case $\Gamma$ is not connected, the complexities of the languages associated with each of its connected components add, the edges being bijectively labeled, and this remains true when each of these components has been contracted to a bouquet of circles. Since if $(a, b) \in Q,\left(a^{\prime}, b^{\prime}\right) \in Q$, then $\left(a+a^{\prime}, b+b^{\prime}\right) \in Q$, the complexity of $L_{0}$ is such that $\left(a_{0}, b_{0}\right) \in Q$ in this case too.

Note that in Cassaigne's Theorem 2.1, the main condition over exact complexities for the $(a, b)$ 's is $2 a+b \leq(a+b)^{2}$, whereas the one in the above proof translates into $2 a+b \leq 3(a+b)-2$ (equivalently, $\mid$ Fact $_{L}(2) \mid=p_{L}(2)=2 a+b \leq 3 m-2=$ $\left.3 p_{L}(1)-2=3(a+b)-2\right)$. Thus: 
Corollary 4.6. There exist infinitely many shifts with exact affine complexity which are not lamination languages.

Proof. The proof of Theorem 2.1 in [7] includes the fact that for each possible exact complexity there is a minimal word $w$ having that complexity. By minimality, $p_{w}$ is equal to the complexity of its shift closure, whence the result.

A carrier graph can be embedded in infinitely many surfaces, and lamination language complexities are thus not related to specific surfaces. Nevertheless for bouquets of circles, a remark can be made: First of all, for the known case, considering a coherent bouquet $\Gamma$ of $m$ circles, a language coding a maximal lamination carried by $\Gamma$ corresponds to the symbolic orbit language of idoc interval exchanges $T$ on $m$ intervals [19], having an exact complexity $a n+b$, with $a=m-1$, $b=1$ [16]. On the associated suspension surface $\Sigma_{T}$ (see Sect. 2.1), the Euler characteristic gives $1-m+C=2-2 g$, where $g$ is the genus of $\Sigma_{T}$, and $C$ is the number of components of its boundary, that is, $a+b=2 g+C-1$. A similar construction of a suspension surface generalizes to non-coherent bouquets of circles, where the central rectangle $R$ of $\Sigma_{T}$ is replaced by a polygon with $2 m$ sides, also foliated, but including singularities. By Proposition 4.1 applied to any bouquet of $m$ circles, the languages coding its maximal carried laminations have an exact complexity $a n+b$, with $a+b=m$, that is, $a+b=2 g+C-1$ too.

\section{Building LAMinations AND LAMinAtion LANGUAGES}

In this section we discuss more constructive methods than the ones behind Lemma 4.4 to build laminations and lamination languages.

\subsection{Complete Complexity families of Lamination languageS}

From Proposition 4.1, one can deduce a criterion to check that a set of curves is a lamination, knowing the complexity of its coding:

Corollary 5.1. Let $\mathcal{C}$ be a set of simple closed or two-way infinite curves in $\Sigma$, all pairwise disjoint and non-homotopic, coded into a shift $L$ by a graph $\Gamma$ labeled by $A$. Then $\mathcal{C}$ is a lamination if $p_{L}(n)=\left(\mid\right.$ Fact $\left._{L}(2)|-| A \mid\right) n+\left(2|A|-\mid\right.$ Fact $\left._{L}(2) \mid\right), \forall n>0$.

Proof. We must check that $\mathcal{C}$ is maximal rel. to some graph. By Lemma 3.2(2), the set $\mathcal{C}$ is carried by $S_{L}(\Gamma)$. Next, if $\mathcal{C}$ was not maximal rel. to $S_{L}(\Gamma)$, curves could be added to $\mathcal{C}$ until it is, while preserving all the properties of the curves, and by Lemma 3.2(3), while also being carried by $\Gamma$. But then, this new set of curves $\mathcal{C}^{\prime}$ is a lamination maximal rel. to $S_{L}(\Gamma)$, and Proposition 4.1 applies to it. Since the added curve codings include distinct factors from the ones in $F_{a c t}$, the complexity of the coding of $\mathcal{C}$ is not equal to the one of $\mathcal{C}^{\prime}$, whence the result. 
Using the preceding result, we now build laminations whose codings have complexities running all the possible exact complexities, that is, we prove Theorem 1.2.

Let $\Gamma_{m}$ be an alternating bouquet of $m$ circles, whose embedding in $\Sigma$ is such that each circle has its two half-edges consecutive around the unique vertex $v$ of $\Gamma_{m}$ with the same orientation order (so that $\Gamma_{m}$ can be embedded as a drawing of a coding carrier graph in a punctured sphere - see the next figures below). Let the edges of $\Gamma_{m}$ be labeled by $A=\left\{a_{1}, \ldots, a_{m}\right\}$, where the $a_{i}$ 's are used in the clockwise order of the circles of $\Gamma_{m}$. If $m=1$, we define the language $L_{1}=\left\{{ }^{\omega} a_{1}^{\omega}\right\}$ which codes the trivial lamination made of a simple closed curve homotopic to the unique circle of $\Gamma_{1}$. If $m>1$ is even, we define the following languages:

$$
\begin{aligned}
& L_{m(\text { with } m \bmod 2 \equiv 0)}=\left\{\omega_{a_{1}} a_{2}^{\omega}\right\} \cup\left\{\omega_{a_{2 i+1}} a_{2 i}^{\omega},{ }^{\omega} a_{2 i+1} a_{2 i+2}^{\omega} \mid i=1, \ldots, \frac{m}{2}-1\right\} \cup \\
& \left\{\omega a_{1} a_{2 i}^{\omega} \mid i=2, \ldots, \frac{m}{2}\right\} \cup \\
& \left\{\omega_{a_{1}} a_{2} a_{3} \ldots a_{2 i}^{\omega} \mid i=2, \ldots, \frac{m}{2}\right\} \cup \\
& \left\{{ }^{\omega} a_{1}\left(a_{2} \ldots a_{m} a_{1}\right)^{\omega}\right\} .
\end{aligned}
$$

For instance, $L_{2}=\left\{{ }^{\omega} a_{1} a_{2}^{\omega},{ }^{\omega} a_{1}\left(a_{2} a_{1}\right)^{\omega}\right\}$, and $L_{6}=\left\{\omega_{a_{1}} a_{2}^{\omega},{ }^{\omega} a_{3} a_{2}^{\omega},{ }^{\omega} a_{3} a_{4}^{\omega},{ }^{\omega} a_{5} a_{4}^{\omega}\right.$, $\left.\omega_{a} a_{6} a_{6}, \quad \omega_{a_{1}} a_{4}^{\omega},{ }^{\omega} a_{1} a_{6}^{\omega}, \quad{ }^{\omega} a_{1} a_{2} a_{3} a_{4}^{\omega}, \quad{ }^{\omega} a_{1} a_{2} a_{3} a_{4} a_{5} a_{6}^{\omega}, \quad{ }^{\omega} a_{1}\left(a_{2} a_{3} a_{4} a_{5} a_{6} a_{1}\right)^{\omega}\right\}$. These words are the codings of curves carried by $\Gamma_{m}$, having their ends spiraling either around single circles of $\Gamma_{m}$, or around the set of all the circles of $\Gamma_{m}$. For instance, here is represented this set of curves carried by $\Gamma_{2}$ coded by $L_{2}$, together with a drawing of the induced Burst $_{v, L_{2}}\left(\Gamma_{2}\right)$ on the right:
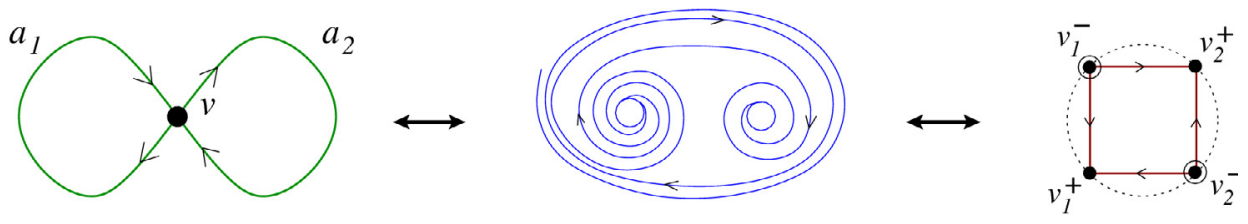

We can also describe more precisely the sets of curves corresponding to the four lines of the definition of $L_{m}$ :

(i) C-shaped double spirals linking neighbor circles, attached together by alternating their orientation, and making a chain around the circles of $\Gamma_{m}$;

(ii) C-shaped double spirals starting from $a_{1}$, linking non-neighbor circles of $\Gamma_{m}$, going within the chain defined in (i);

(iii) C-shaped double spirals starting from $a_{1}$, linking non-neighbor circles of $\Gamma_{m}$, going externally to the chain defined in (i);

(iv) a double spiral starting from $a_{1}$ and then spiraling globally around $\Gamma_{m}$.

For instance, here are the curves carried by $\Gamma_{6}$ coded by $L_{6}$, shown by a union of two sets of curves, the first one corresponding to the curves of kind (i) and (ii), 
and the second one to the curves of kind (iii) and (iv), together with a drawing of the induced Burst $_{v, L_{6}}\left(\Gamma_{6}\right)$ on the right:

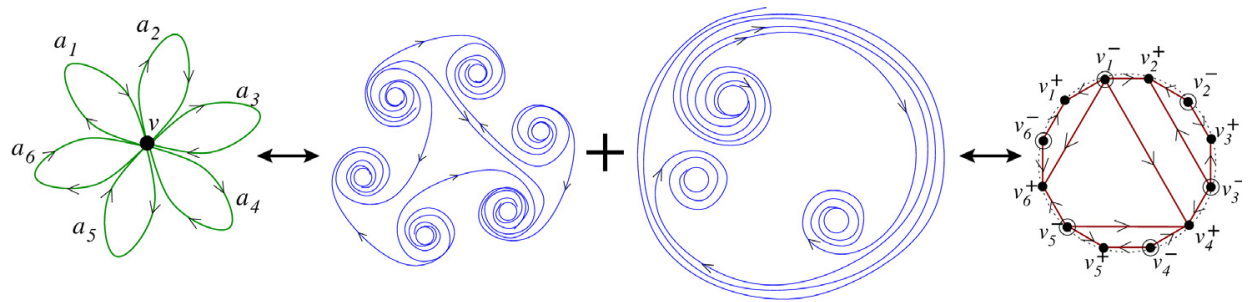

If $m>1$ is odd, we define the following languages similarly to the even case:

$L_{m(\text { with } m \bmod 2 \equiv 1)}=$

$$
\begin{aligned}
& \left\{{ }^{\omega} a_{1} a_{2}^{\omega}\right\} \cup\left\{{ }^{\omega} a_{2 i+1} a_{2 i}^{\omega},{ }^{\omega} a_{2 i+1} a_{2 i+2}^{\omega} \mid i=1, \ldots, \frac{m-1}{2}-1\right\} \cup\left\{{ }^{\omega} a_{m} a_{m-1}^{\omega}\right\} \cup \\
& \left\{{ }^{\omega} a_{1} a_{2 i}^{\omega} \mid i=2, \ldots, \frac{m-1}{2}\right\} \cup \\
& \left\{{ }^{\omega} a_{1} a_{2} a_{3} \ldots a_{2 i}^{\omega} \mid i=2, \ldots, \frac{m-1}{2}\right\} \cup\left\{{ }^{\omega} a_{1} \ldots a_{m} a_{m-1}^{\omega}\right\} \cup \\
& \left\{{ }^{\omega} a_{1}\left(a_{2} \ldots a_{m} a_{1}\right)^{\omega}\right\} .
\end{aligned}
$$

For instance, $L_{7}=\left\{{ }^{\omega} a_{1} a_{2}^{\omega},{ }^{\omega} a_{3} a_{2}^{\omega},{ }^{\omega} a_{3} a_{4}^{\omega},{ }^{\omega} a_{5} a_{4}^{\omega},{ }^{\omega} a_{5} a_{6}^{\omega},{ }^{\omega} a_{7} a_{6}^{\omega},{ }^{\omega} a_{1} a_{4}^{\omega},{ }^{\omega} a_{1} a_{6}^{\omega}\right.$, $\left.\omega_{a_{1}} a_{2} a_{3} a_{4}^{\omega},{ }^{\omega} a_{1} a_{2} a_{3} a_{4} a_{5} a_{6}^{\omega},{ }^{\omega} a_{1} a_{2} a_{3} a_{4} a_{5} a_{6} a_{7} a_{6}^{\omega},{ }^{\omega} a_{1}\left(a_{2} a_{3} a_{4} a_{5} a_{6} a_{7} a_{1}\right)^{\omega}\right\}$. Here are represented the corresponding curves carried by $\Gamma_{7}$ and coded by $L_{7}$, together with a drawing of the induced Burst $_{v, L_{7}}\left(\Gamma_{7}\right)$ on the right:

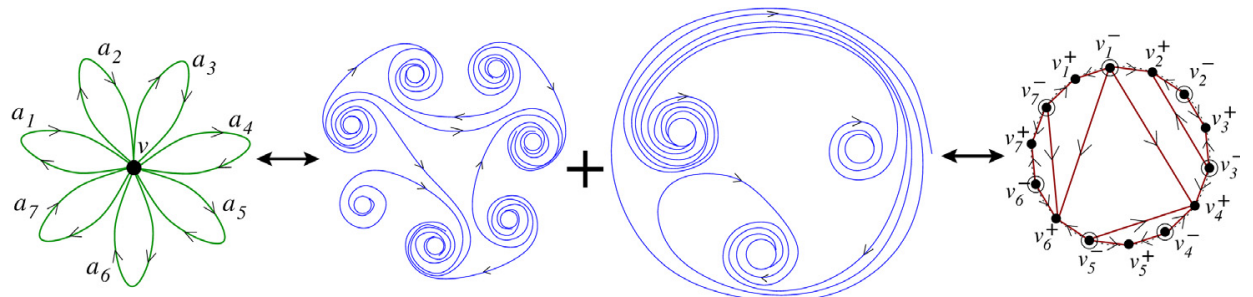

Now, the shift orbit closure $L_{m}^{\sigma}$ of $L_{m}$ for every $m>0$ includes all the shifted words in $L_{m}$, and also the shifted periodic words in $\left\{\omega_{a}^{\omega} \mid i=1 \ldots m\right\} \cup$ ${ }^{\omega}\left(a_{1} \ldots a_{m}\right)^{\omega}$, that is, the only two-way infinite limit words to be added, and corresponding to the left or right periodicities of the words in $L_{m}$. In terms of curves, these periodic words are the codings of the closed curves which are the limits of the spiraling ends of the curves coded by $L_{m}$. Let $\mathcal{L}_{m}$ denote the set of curves coded by $L_{m}^{\sigma}$ :

Lemma 5.2. For every $m>0, \mathcal{L}_{m}$ is a lamination coded into $L_{m}^{\sigma}$ by $\Gamma_{m}$, for which $p_{L_{m}^{\sigma}}(n)=(2 m-2) n+(2-m), \forall n>0$. 
Proof. By construction, for every $m>0$, the curves of $\mathcal{L}_{m}$ are simple, closed or two-way infinite, pairwise disjoint, pairwise non-homotopic, all carried by $\Gamma_{m}$, and thus by $S_{L_{m}^{\sigma}}\left(\Gamma_{m}\right)$ too. Also, when $m$ is even, we have:

$$
\begin{aligned}
\text { Fact }_{L_{m}^{\sigma}}(2)= & \left\{a_{i}^{2} \mid i=1 \ldots m\right\} \cup\left\{a_{2 i+1} a_{2 i} \mid i=1, \ldots, \frac{m}{2}-1\right\} \cup \\
& \left\{a_{1} a_{2 i} \mid i=2, \ldots, \frac{m}{2}\right\} \cup\left\{a_{i} a_{i+1} \mid i=1, \ldots, m-1\right\} \cup\left\{a_{m} a_{1}\right\} .
\end{aligned}
$$

And when $m$ is odd:

$$
\begin{aligned}
\text { Fact }_{L_{m}^{\sigma}}(2)= & \left\{a_{i}^{2} \mid i=1 \ldots m\right\} \cup\left\{a_{2 i+1} a_{2 i} \mid i=1, \ldots, \frac{m-1}{2}\right\} \cup \\
& \left\{a_{1} a_{2 i} \mid i=2, \ldots, \frac{m-1}{2}\right\} \cup\left\{a_{i} a_{i+1} \mid i=1 \ldots m-1\right\} \cup\left\{a_{m} a_{1}\right\} .
\end{aligned}
$$

For every $m>0, \mid$ Fact $_{L_{m}^{\sigma}}(2) \mid=3 m-2$, and thus by Corollary $5.1, \mathcal{L}_{m}$ is a lamination if $p_{L_{m}^{\sigma}}(n)=(2 m-2) n+(2-m), \forall n>0$. Note that $p_{L_{m}^{\sigma}} \equiv p_{L_{m}}$, and that $L_{m}^{\sigma}$ involves only ultimately periodic words, i.e. words ${ }^{\omega} v_{1} u v_{2}^{\omega}$, where $v_{1}, u, v_{2}$ are finite words, $u$ being possibly the empty word (periodic words are included in this definition when $v_{1}=v_{2}$ and $u$ is empty). We then check the above complexities for the $L_{m}$ 's by first considering the following fact: a word $w={ }^{\omega} a_{i} a_{j}^{\omega}$, with $a_{i}, a_{j} \in A$ and $a_{i} \neq a_{j}$, has complexity $p_{w}(n)=n+1, \forall n>0$ (words of this form are skew Sturmian words [23]). Now, if $L=\left\{\omega_{a} a_{j}^{\omega}, \omega_{a_{k}} a_{l}^{\omega}\right\}$ with $a_{i}, a_{j}, a_{k}, a_{l} \in A, a_{i} a_{j} \neq a_{k} a_{l}, a_{i} \neq a_{j}$ and $a_{k} \neq a_{l}$, then $p_{L}(n)=2(n+1)-(4-t)$, $\forall n>0$, where $t$ is the number of distinct letters among $a_{i}, a_{j}, a_{k}, a_{l}$. Indeed, in ${ }^{\omega} a_{i} a_{j}^{\omega}$ and ${ }^{\omega} a_{k} a_{l}^{\omega}$, if two of their letters are equal, say to $a$, the only factors in common are $a^{n}$ for each $n>0$. For instance, if $L=\left\{{ }^{\omega} a b^{\omega},{ }^{\omega} b a^{\omega}\right\}$, then $p_{L}(n)=2 n$, $\forall n>0$. From this complexity rule, when $m$ is even, the complexity of $L_{m}$ is obtained as follows (it is obtained similarly when $m$ is odd):

- The subset $H_{1}=\left\{{ }^{\omega} a_{1} a_{2}^{\omega}\right\} \cup\left\{{ }^{\omega} a_{2 i+1} a_{2 i}^{\omega}, \omega_{a_{2 i+1}} a_{2 i+2}^{\omega} \mid i=1, \ldots, \frac{m}{2}-1\right\}$ has complexity $q_{1}(n)=p_{H_{1}}(n)=(m-1)(n+1)-(m-2)=(m-1) n+1, \forall n>0$, since adding one by one the complexities of these $m-1$ words means to apply $m-2$ times the rule above with $t=3$.

- Adding $H_{2}=\left\{{ }^{\omega} a_{1} a_{2 i}^{\omega} \mid i=2, \ldots, \frac{m}{2}\right\}$ means to add to $p_{H_{1}}(n)$ the function $q_{2}(n)=\left(\frac{m}{2}-1\right)(n+1)-2\left(\frac{m}{2}-1\right)=\left(\frac{m}{2}-1\right) n-\left(\frac{m}{2}-1\right), \forall n>0$, since adding one by one the complexities of these $\frac{m}{2}-1$ words means to apply $\frac{m}{2}-1$ times the rule above with $t=2$ (these words have their two letters in common with the others in $H_{1}$ ).

- For $H_{3}=\left\{{ }^{\omega} a_{1} a_{2} a_{3} \ldots a_{2 i}^{\omega} \mid i=2, \ldots, \frac{m}{2}\right\}$, when adding one by one its words to $H_{1} \cup H_{2}$, we see that at each adding the only new length-2 factor is $a_{2 j} a_{2 j+1}$ with $j=i-1$, and thus for each length $n>2$, the new factors are those containing $a_{2 j} a_{2 j+1}$, that is, $n-1$ factors. Hence the complexity contribution of $H_{3}$ is $q_{3}(n)=\left(\frac{m}{2}-1\right)(n-1), \forall n>0$. 
- Finally, $H_{4}=\left\{a_{1}\left(a_{2} \ldots a_{m} a_{1}\right)^{\omega}\right\}$ is made of a word with the same property as the ones in $H_{3}$ where the only new length-2 factor is $a_{m} a_{1}$, so that the contribution of $H_{4}$ is $q_{4}(n)=n-1, \forall n>0$.

Hence $p_{L_{m}}(n)=\sum_{i=1 . .4} q_{i}(n)=((m-1) n+1)+\left(\left(\frac{m}{2}-1\right) n-\left(\frac{m}{2}-1\right)\right)+$ $\left(\left(\frac{m}{2}-1\right)(n-1)\right)+(n-1)=(2 m-2) n+(2-m)$, thus $\mathcal{L}_{m}$ is a lamination.

For instance, with respect to the examples of the preceding figures, $L_{6}^{\sigma}$ is a lamination language with complexity $p_{L_{6}^{\sigma}}(n)=10 n-4$, and $L_{7}^{\sigma}$ has complexity $p_{L_{7}^{\sigma}}(n)=12 n-5, \forall n>0$.

Now, the exact complexities $a n+b$ of the $L_{m}^{\sigma}$ 's, are such that each $(a, b)=$ $(2 m-2,2-m)$ is on the lower boundary of the region $Q \subset \mathbb{N} \times \mathbb{Z}$ of the possible complexities given by Theorem 1.1, with the additional property that there is no $\left(a^{\prime}, b^{\prime}\right) \in Q$, with $a^{\prime}<a$ or $b^{\prime}<b$. These languages have these extremal complexities because for every $m>0,\left|F_{a c t} t_{m}^{\sigma}(2)\right|=3 m-2$, which is the maximal possible value associated with the number of edges of $B u r s t_{v, L_{m}^{\sigma}}$, that is of $S_{L_{m}^{\sigma}}\left(\Gamma_{m}\right)$ too, for an alternating bouquet of circles (see Cor. 3.6).

In order to get the other $(a, b)$ 's of the lower boundary of $Q$, that is, the complexities for which $a$ is odd, and for which there is no point $\left(a^{\prime}, b^{\prime}\right) \in Q$, with $b^{\prime}<b$, it is sufficient to have the preceding bursts with one edge removed. Indeed, this removal is equivalent to having one factor less in Fact(2), that is, $3 m-3$ of them, so that by Proposition 4.1 the corresponding languages would have complexity $(2 m-3) n+(3-m)$. Thus, for $m=2$, we define $L_{2}^{\prime}=L_{2} \backslash\left\{{ }^{\omega} a_{1}\left(a_{2} a_{1}\right)^{\omega}\right\}$, removing only the factor $a_{2} a_{1}$ from $\operatorname{Fact}_{L_{2}^{\sigma}}(2)$, contained only in ${ }^{\omega} a_{1}\left(a_{2} a_{1}\right)^{\omega}$. Then $L_{2}^{\prime}$ consists of a single skew Sturmian word, and $p_{L^{\prime}{ }_{2}}(n)=p_{L_{2}^{\prime}}(n)=n+1, \forall n>0$. For $m>2$, we define $L_{m}^{\prime}=L_{m} \backslash\left\{\omega_{a} a_{3} a_{2}^{\omega}\right\}$, removing only the factor $a_{3} a_{2}$ from Fact $_{L_{m}^{\sigma}}(2)$, contained only in $\omega_{a_{3}} a_{2}^{\omega}$. From the proof of Lemma 5.2, we see that removing $\omega_{a} a_{2}^{\omega}$ means to subtract an $(n-1)$ contribution to the complexity of $L_{m}$, that is, $p_{L^{\prime \sigma}}(n)=p_{L_{m}^{\prime}}(n)=p_{L_{m}}(n)-(n-1)=(2 m-3) n+(2-m+1)$, $\forall n>0$. Hence the complexities of the $L_{m}^{\prime \sigma}$ 's are the ones expected to apply Corollary 5.1, thus if $\gamma_{m}$ is the curve corresponding to the removed word, maximality of $\mathcal{L}_{m}^{\prime}=\mathcal{L}_{m}-\left\{\gamma_{m}\right\}$ rel. to $S_{L^{\prime \sigma}{ }_{m}}\left(\Gamma_{m}\right)$ holds, and $\mathcal{L}_{m}^{\prime}$ is a lamination. For instance, for $L_{6}^{\prime \sigma}$, then $p_{L_{6}^{\prime \sigma}}(n)=9 n-3$, and for $L_{7}^{\prime \sigma}$, then $p_{L_{7}^{\prime \sigma}}(n)=11 n-4, \forall n>0$.

As a result, we have described lamination languages with exact complexities covering all the pairs $(a, b)$ of the lower boundary of $Q$. In order to obtain the other pairs, we use another simple graph move called edge subdivision: let $e$ be any edge of a graph $\Gamma$, then the subdivision of $e$ consists in putting a new vertex $v$ in $e$, dividing it into two edges so that $v$ has degree 2. For instance:
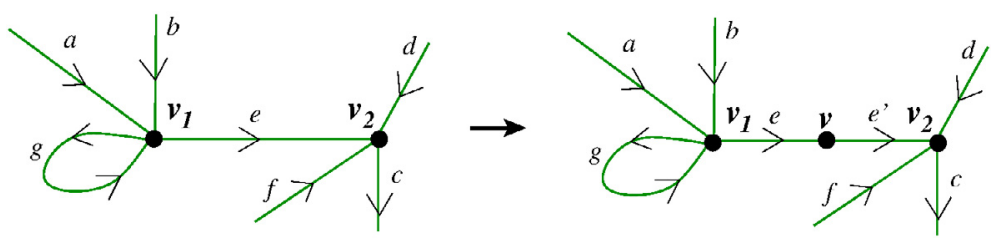
Lemma 5.3. Let $\mathcal{L}$ be a lamination coded by $\Gamma$ into $L$, and maximal rel. to $S_{L}(\Gamma)$. Let $\Gamma^{\prime}$ be $\Gamma$ to which one edge subdivision has been applied, and let $L^{\prime}$ be the coding of $\mathcal{L}$ by $\Gamma^{\prime}$. Then $\mathcal{L}$ is also maximal rel. to $S_{L^{\prime}}\left(\Gamma^{\prime}\right)$.

Proof. A subdivision of an edge $e$ in $\Gamma$ means to replace the corresponding vertex $v_{e}$ in $S_{L}(\Gamma)$ by an edge in $S_{L^{\prime}}\left(\Gamma^{\prime}\right)$. By Lemma 3.1(2), $v_{e}$ is coherent, thus this replacement has no effect on the carrying possibilities, whence the result.

Lemma 5.4. Let $L$ be a lamination language coding a lamination $\mathcal{L}$ by a graph $\Gamma$, $\mathcal{L}$ being maximal rel. to $S_{L}(\Gamma)$. Let $\Gamma^{\prime}$ be $\Gamma$ to which one edge subdivision has been applied, and let $L^{\prime}$ be the coding of $\mathcal{L}$ carried by $\Gamma^{\prime}$. Then, $p_{L^{\prime}}=p_{L}(n)+1, \forall n>0$.

Proof. According to Lemma 5.3 and its proof, one can apply Proposition 4.1 to compute $p_{L^{\prime}}$ with $\mid$ Fact $_{L^{\prime}}(2)|=| F_{\text {act }}(2) \mid+1$, and with an alphabet $A^{\prime}$ such that $\left|A^{\prime}\right|=|A|+1$.

Now, by applying Lemma 5.4 to each $L_{m}^{\sigma}$ and $L_{m}^{\prime \sigma}$, that is, by subdividing the edges of their corresponding bouquets of circles $\Gamma_{m}$, we can arbitrarily increment the $b$ part of their exact complexities $a n+b$, and get lamination languages having complexities covering all the region $Q$. Moreover, the corresponding laminations $\mathcal{L}_{m}, \mathcal{L}_{m}^{\prime}$ are finite, and they are connected sets, since for each circle of $\Gamma_{m}$ there is a curve with an end spiraling around it, and any two circles are joined by a chain of such curves. Hence the proof of Theorem 1.2 is complete.

Note that the above construction was based on specific embeddings of alternating bouquets of circles $\Gamma_{m}$, and on a specific family of laminations $\mathcal{L}_{m}, \mathcal{L}_{m}^{\prime}$ carried by them. There are other possible embeddings for such bouquets of circles and other finite carried laminations. We could also drop the idea of describing a complete family for every possible complexity, and consider bouquets of circles which are not alternating. For instance, here are two such embedded bouquets carrying maximal laminations [14]:
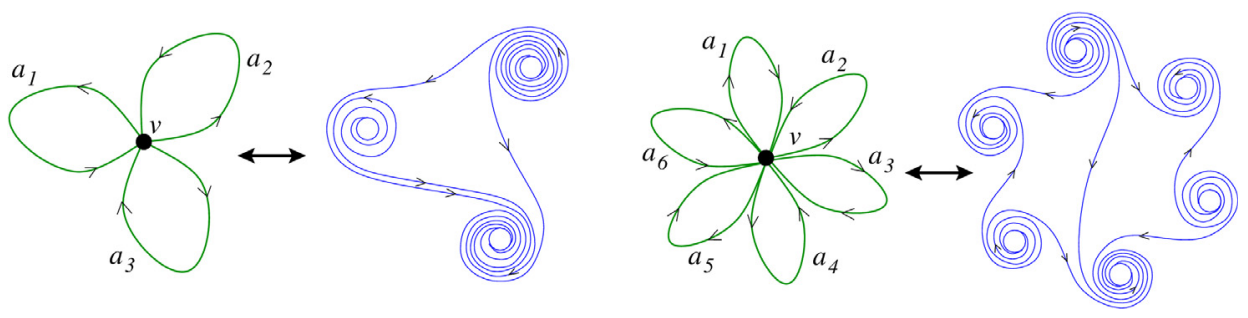

For the carried lamination on the left, its coding lamination language $L$ is such that $\operatorname{Fact}_{L}(2)=\left\{a_{i}^{2} \mid i=1,2,3\right\} \cup\left\{a_{1} a_{3}, a_{2} a_{3}, a_{2} a_{1}\right\}$, thus $p_{L}(n)=3 n, \forall n>$ 0 . For the one on the right, $L$ is such that $\operatorname{Fact}_{L}(2)=\left\{a_{i}^{2} \mid i=1 \ldots 6\right\} \cup$ $\left\{a_{1} a_{2}, a_{3} a_{2}, a_{3} a_{4}, a_{5} a_{4}, a_{5} a_{6}, a_{1} a_{6}, a_{1} a_{4}\right\}$, thus $p_{L}(n)=7 n-1, \forall n>0$. 


\subsection{Some Minimal LAMinAtion LANGUAGES}

In the preceding section, the lamination languages allowing us to cover all the possible exact complexities given by Theorem 1.1 were finite and made of ultimately periodic words. For aperiodic minimal lamination languages, we still do not know about a fully constructive method to build such a family. A step towards a solution would be to use the relationship between laminations carried by coherent bouquets of circles and interval exchanges (see Sect. 2.1). There are indeed ways to generate the symbolic orbits of interval exchanges, corresponding then to lamination languages. These techniques are e.g. based on Rauzy induction $[3,12]$ and on substitution compositions. A substitution is indeed a simple rewriting rule defined by a map $\theta: A \rightarrow B^{*}$, where $A, B$ are finite alphabets, $B^{*}$ denotes the set of finite words over $B$, which extends to all words by sending $w=\ldots a_{n} a_{n+1} a_{n+2} \ldots$ to $\theta(w)=\ldots \theta\left(a_{n}\right) \theta\left(a_{n+1}\right) \theta\left(a_{n+2}\right) \ldots$

Here however we focus on another construction able to produce minimal lamination languages associated sometimes with non-coherent bouquets of circles, and relying on substitution iterations and letter projections only. This construction is derived from the fact that some pseudo-Anosov diffeomorphisms of surfaces, i.e. diffeomorphisms which always have one stable and one unstable minimal laminations [24,29], can be represented by substitutions in the symbolic domain $[18,19]$. By iterating these substitutions, it is then possible to obtain the lamination languages which code their associated stable laminations. Let us here recall this technique in a simplified setting from [19]: A directed graph $\Gamma=(V, E)$ is said to be cycle-based if it is strongly connected and if it can be described as the union of $k+h=n$ oriented cycles $\left\{C_{1}, \ldots, C_{n}\right\}$ as follows: (i) $\left\{C_{1}, \ldots, C_{k}\right\}$ is a set of pairwise disjoint cycles with respective non-empty finite sets of vertices $V_{i}$, such that $V=\bigcup_{i=1}^{k} V_{i}$; (ii) $\pi$ is a permutation over $V$ such that $v \in V$ is linked to $\pi(v)$ by an edge in $E$ not in $\left\{C_{1}, \ldots, C_{k}\right\}$, thus determining the other cycles $\left\{C_{k+1}, \ldots, C_{k+h}\right\}$.

Example 5.5. Let $C_{1}, C_{2}$ be two cycles with two vertices each, respectively $v_{1}, v_{2}$ and $v_{3}, v_{4}$, and let $\pi=\left(v_{1} v_{3}\right)\left(v_{2} v_{4}\right)$ inducing two other cycles $C_{3}, C_{4}$. The result is the following cycle-based graph:

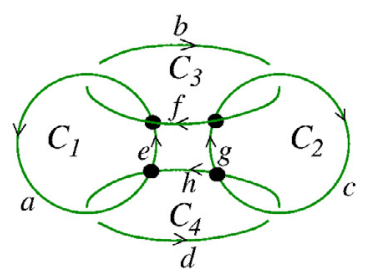

Now, let $\Gamma$ be a cycle-based graph labeled by $A$, embedded in a surface $\Sigma$ as a coding carrier graph, with the following constraints: at each vertex of $\Gamma$, the crossing orientation - by construction, this crossing is made of exactly two cycles of $\Gamma$ - must be consistent with the others, that is, the relative orientations of the 
edges at each crossing must match when translated along any edge path of $\Gamma$ (see e.g. the figure above). Now let $c_{i}^{(v)}$ denote the finite path label of the cycle $C_{i}$ of $\Gamma$ starting from the vertex $v \in C_{i}$. Then we associate a substitution $\theta_{i}$ with $C_{i}$, defined as the identity over all the letters in $A$, except for the letters $x_{i}^{(v)}$ for which $\theta_{i}\left(x_{i}^{(v)}\right)=x_{i}^{(v)} c_{i}^{(v)}$, where $v$ is any vertex of $C_{i}$, and where $x_{i}^{(v)}$ is the label of the edge of $\Gamma$ whose one of the half-edges is incoming at $v$ while not being in $C_{i}$. We denote by $\mathcal{T}_{\Gamma}$ the set of the $n$ substitutions $\theta_{i}$ over $A$ associated with the $n$ cycles of $\Gamma$. For instance, the four substitutions of $\mathcal{T}_{\Gamma}$ where $\Gamma$ is the graph of Example 5.5 are the following (we only give the images of the letters which are not the identity):

$$
\begin{aligned}
& \theta_{1}(f)=\text { fae } \theta_{2}(b)=b c g \theta_{3}(e)=e b f \theta_{4}(a)=a d h \\
& \theta_{1}(h)=\text { hea } \theta_{2}(d)=d g c \theta_{3}(g)=g f b \theta_{4}(c)=c h d
\end{aligned}
$$

Substitutions as above are said to be non-erasing, i.e. there is no letter whose image is the empty word. A word $w$ is a fixed point of a substitution $\theta$, if $\theta(w)=w$. One-way right infinite fixed points can be obtained by iterating $\theta(a)$, whenever $\theta$ is non-erasing, and $a \in A$ is a strict prefix of $\theta(a)$, so that for every $n>0, \theta^{n}(a)$ is a strict prefix of $\theta^{(n+1)}(a)$. When such a one-way infinite word $w$ is minimal, its associated shift orbit closure $L_{w}^{\sigma}$ in $A^{\mathbb{Z}}$ is defined as the set of the two-way infinite words whose factor set is Fact $_{w}$, and accordingly $w$ is sufficient to study the combinatorics of $L_{w}^{\sigma}$ (see also Sect. 2.3).

Theorem 5.6. (see [18]). Let $\Gamma$ be a cycle-based graph labeled by A. Let $\theta$ be a finite composition of substitutions in $\mathcal{T}_{\Gamma}$, where each $\theta_{i} \in \mathcal{T}_{\Gamma}$ occurs at least once. Then there is a letter $a \in A$, such that iterating $\theta(a)$ gives a fixed point $w$ which is minimal and which codes a half-curve of a maximal lamination $\mathcal{L}$ rel. to $\Gamma$, where $L_{w}^{\sigma}$ is the lamination language coding $\mathcal{L}$ by $\Gamma$.

Corollary 5.7 (see [19], 5.3.2). Let $L$ be a lamination language obtained by Theorem 5.6 from a cycle-based graph $\Gamma=(V, E)$. Then the complexity of $L$ is $p_{L}(n)=|V| n+|V|, \forall n>0$.

Proof. As an embedded cycle-based graph, $\Gamma$ is coherent, and it is such that $|E|=2|V|$ with $\partial^{-}(v)=\partial^{+}(v)=2$, for every $v \in V$. Moreover, $\mathcal{L}$ is maximal rel. to $\Gamma$. Thus according to Lemma 3.5, when constructing $S_{L}(\Gamma)$, each burst is maximally biplanar, hence generates three edges, so that $\left|F_{a c t}(2)\right|=3|V|$. By Proposition 4.1, the result follows.

Thus for instance considering the substitutions associated with Example 5.5, a fixed point of any composition of the $\theta_{i}$ 's involving each $\theta_{i}$ at least once, is a minimal lamination half-word $w$ with complexity $p_{w}(n)=4 n+4, \forall n>0$, e.g. $\left(\theta_{1} \theta_{2} \theta_{3} \theta_{4}\right)^{\omega}(a)=$ adgcheadgcgfaebc $\ldots$

Cycle-based graphs are not bouquets of circles, except in the case of two circles built from a cycle $C_{1}$ with one vertex and a trivial $\pi$ generating another cycle $C_{2}$ 
(this case corresponds to interval exchanges over two intervals, and thus to the Sturmian case). However, bouquets of circles can be obtained by applying edge contractions, similarly to what has been done in the proof of Theorem 1.1. Now, symbolically, an edge contraction has the trivial effect of erasing the letter labeling the contracted edge. With this respect, let eras $_{A^{\prime}}$ denote the erasing substitution (or letter projection) over $A$ which is the identity except for the letters in $A^{\prime} \subset A$ which are sent to the empty word. If $L$ is a shift in $A^{\mathbb{Z}}$, and $\theta$ is a substitution over $A$, then $\theta(L)$ denotes the shift orbit closure of $\{\theta(w) \mid w \in L\}$ :

Corollary 5.8. Let $\mathcal{L}$ be a lamination obtained by Theorem 5.6 from a cycle-based graph $\Gamma=(V, E)$ labeled by $A$. Let $L$ be the lamination language coding $\mathcal{L}$ by $\Gamma$. Let $\Gamma^{\prime}$ be a bouquet of circles obtained by iteratively contracting edges of $\Gamma$, and let $L^{\prime}$ be the coding of $\mathcal{L}$ by $\Gamma^{\prime}$, which is such that $L^{\prime}=\operatorname{eras}_{A^{\prime}}(L)$ where $A^{\prime} \subset A$ is the set of labels of the contracted edges. Then $p_{L^{\prime}}(n)=|V| n+1, \forall n>0$.

Proof. A cycle-based graph is assumed connected, thus $\Gamma$ can be contracted into a bouquet of circles with $|V|-1$ edge contractions. Hence, by Corollary 5.7, and next by iteratively applying Lemma 4.3 , the result follows.

For instance, considering the graph $\Gamma$ of Example 5.5, we can contract three of its edges to obtain a non-coherent bouquet of five circles as follows:
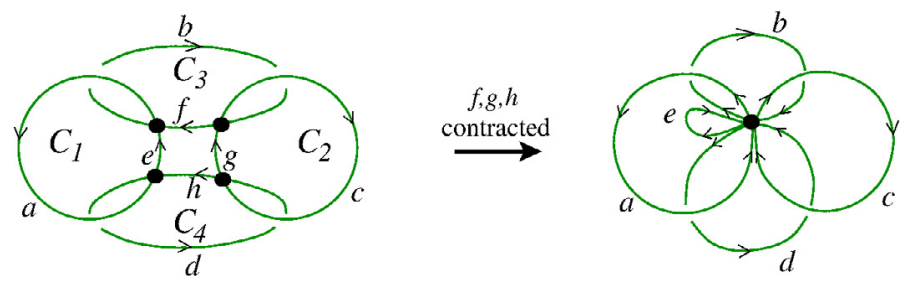

Then according to Corollary 5.8, iterating a composition of the associated four substitutions $\theta_{i}$ of $\mathcal{T}_{\Gamma}$, and applying $\operatorname{eras}_{\{f, g, h\}}$, we get minimal words of complexity $p(n)=4 n+1, \forall n>0$ (e.g. $\left.\operatorname{eras}_{\{f, g, h\}}\left(\left(\theta_{1} \theta_{2} \theta_{3} \theta_{4}\right)^{\omega}\right)(a)\right)$, which are minimal lamination half-words too.

Here is another example illustrating all the above generation steps. Let $\Gamma$ be a graph based on six cycles with nine vertices, and where eight edges are contracted to obtain a non-coherent bouquet of ten circles:
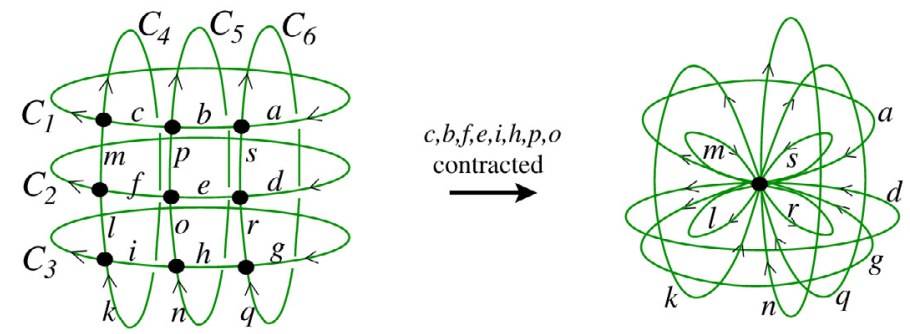
Then, the six associated substitutions of $\mathcal{T}_{\Gamma}$ are the following (we only give the images of the letters which are not the identity):

$$
\begin{array}{lll}
\theta_{1}(m)=\text { mabc } & \theta_{2}(l)=\text { ldef } & \theta_{3}(k)=\text { kghi } \\
\theta_{1}(p)=\text { pcab } & \theta_{2}(o)=\text { ofde } & \theta_{3}(n)=\text { nigh } \\
\theta_{1}(s)=\text { sbca } & \theta_{2}(r)=\text { refd } & \theta_{3}(q)=\text { qhig } \\
\theta_{4}(c)=\text { cklm } & \theta_{5}(b)=\text { bnop } & \theta_{6}(a)=\text { aqrs } \\
\theta_{4}(f)=\text { fmkl } & \theta_{5}(e)=\text { epno } & \theta_{6}(d)=\text { dsqr } \\
\theta_{4}(i)=\text { llmk } & \theta_{5}(h)=\text { hopn } & \theta_{6}(g)=\text { grsq }
\end{array}
$$

The complexity of the fixed point words obtained by iterating compositions of these substitutions involving each $\theta_{i}$ at least once is $p(n)=9 n+9, \forall n>0$, and after erasing the eight letters corresponding to the contracted edges, it becomes $p(n)=$ $9 n+1, \forall n>0$, as is the complexity of the corresponding lamination languages.

The above generation technique produces lamination languages as shift orbit closures of minimal words which are fixed points of a single substitution $\theta$, to which a second substitution of type eras is applied. This kind of words are well-known and called substitutive (or morphic) [2,8]. However in terms of all the possible complexities given by Theorem 1.1, this technique does not cover all of them: cycle-based graphs exist with any number of vertices, so as to give by Corollary 5.8 languages of exact complexities $a n+b$, with $a \geq 1, b=1$, associated with bouquets of circles. Next, by applying edge subdivisions together with Lemma 5.4, we cover every $b \geq 1$, but not the complexities for which $b<1$.

Note also that the above examples of contracted cycle-based graphs yield non-coherent bouquets of circles, while producing lamination languages with exact complexity of the form an +1 , that is, languages with complexity of the same form as the natural symbolic orbit languages of idoc interval exchanges. However, one can prove e.g. by using the explicit characterizations of these interval exchange languages $[4,11]$, that for instance the projected fixed point $\operatorname{eras}_{\{f, g, h\}}\left(\left(\theta_{1} \theta_{2} \theta_{3} \theta_{4}\right)^{\omega}\right)(a)$ does not occur in one of them as a half-word, and thus that the corresponding lamination language is not an interval exchange language.

As a final remark, let us sum up some of the problems which remain to be solved in the context of this paper: understanding the characteristics of all the lamination languages having exact complexities of the form $a n+1$, finding constructive families of aperiodic minimal lamination languages covering every possible exact complexity, enumerating finite laminations within the framework used in Section 5.1, analyzing from a word-combinatorics viewpoint the geometric constraints which lead to Corollary 4.6, characterizing the possible non-exact affine complexities of lamination languages. 


\section{REFERENCES}

[1] R.L. Adler, A.G. Konheim and M.H. McAndrew, Topological entropy. Trans. Amer. Math. Soc. 114 (1965) 309-319.

[2] J-P. Allouche and J. Shallit, Automatic sequences. Cambridge University Press, Cambridge (2003).

[3] P. Arnoux and G. Rauzy, Représentation géométrique de suites de complexité $2 n+1$. Bull. Soc. Math. France 119 (1991) 199-215.

[4] A. Ya. Belov and A.L. Chernyatiev, Words with low complexity and interval exchange transformations. Commun. Moscow Math. Soc. 63 (2008) 159-160.

[5] F. Bonahon, Geodesic laminations on surfaces. In Laminations and foliations in dynamics, geometry and topology, vol. 269 of Contemp. Math. Amer. Math. Soc. (2001) 1-37.

[6] D. Calegari, Foliations and the geometry of 3-manifolds. Oxford Mathematical Monographs. Oxford University Press, Oxford (2007).

[7] J. Cassaigne, Complexité et facteurs spéciaux. Bull. Belg. Math. Soc. 1 (1997) 67-88.

[8] J. Cassaigne and F. Nicolas, Factor complexity, Combinatorics, automata and number theory, vol. 135 of Encyclopedia Math. Appl. Cambridge University Press, Cambridge (2010) $163-247$.

[9] A.J. Casson and S. Bleiler, Automorphisms of surfaces after Nielsen and Thurston, vol. 9 of London Mathematical Society Student Texts. Cambridge University Press, Cambridge (1988).

[10] V. Dujmović et al., A fixed-parameter approach to 2-layer planarization. Algorithmica 45 (2006) 159-182.

[11] S. Ferenczi and L. Q. Zamboni Languages of $k$-interval exchange transformations. Bull. Lond. Math. Soc. 40 (2008) 705-714.

[12] N. Pytheas Fogg, Substitutions in dynamics, arithmetics and combinatorics, vol. 1794 of Lect. Notes Math. Edited by V. Berthé, S. Ferenczi, C. Mauduit and A. Siegel. Springer, Verlag, Berlin (2002).

[13] R.K. Guy, Outerthickness and outercoarseness of graphs, Combinatorics in Proc. British Combinatorial Conf., Univ. Coll. Wales, Aberystwyth, 1973. London Math. Soc. Lect. Note Ser. Cambridge University Press, London (1974) 57-60.

[14] I. Hargittai and C.A. Pickover, Spiral Symmetry. World Scientific (1992).

[15] A.E. Hatcher, Measured lamination spaces for surfaces, from the topological viewpoint. Topology Appl. 30 (198)8 63-88.

[16] M. Keane, Interval exchange transformations. Math. Z. 141 (1975) 25-31.

[17] D. Lind and B. Marcus, Symbolic Dynamics and Coding. Cambridge University Press, Cambridge (1995).

[18] L.-M. Lopez and Ph. Narbel, Languages, D0L-systems, sets of curves, and surface automorphisms. Inform. Comput. 180 (2003) 30-52.

[19] L.-M. Lopez and Ph. Narbel, Lamination languages. Ergodic Theory Dynam. Systems 33 (2013) 1813-1863.

[20] M. Lothaire, Combinatorics on Words, number 17 in Encyclopedia of Math. Appl. Cambridge University Press, Cambridge (1997).

[21] R. Mañé, Ergodic Theory and Differentiable Dynamics. Springer-Verlag, Berlin (1987).

[22] M. Morse and G.A. Hedlund, Symbolic dynamics I. Amer. J. Math. 60 (1938) 815-866.

[23] M. Morse and G.A. Hedlund, Symbolic dynamics II. Sturmian trajectories. Amer. J. Math. 62 (1940) $1-42$.

[24] R.C. Penner, A construction of pseudo-Anosov homeomorphisms. Trans. Amer. Math. Soc. 310 (1988) 179-197.

[25] R.C. Penner and J.L. Harer, Combinatorics of train tracks, vol. 125 of Annal. Math. Studies. Princeton University Press, Princeton, NJ (1992).

[26] D. Perrin and J.P. Pin, Infinite Words, number 141 in Pure Appl. Math. Elsevier (2004). 
[27] M. Quéffelec, Substitution dynamical systems-spectral analysis, 2nd Edition. Vol. 1294 of Lect. Notes Math. Springer-Verlag, Berlin (2010).

[28] W.P. Thurston, The geometry and topology of three-manifolds. Princeton University Lecture Notes (Electronic version 1.1, 2002). http://library.msri.org/books/gt3m (1980).

[29] W.P. Thurston, On the geometry and dynamics of diffeomorphisms of surfaces. Bull. Amer. Math. Soc. 19 (1988) 417-431.

Communicated by Ch. Choffrut.

Received March 14, 2014. Accepted March 17, 2014. 